\title{
Maastrichtian-paleogene benthic foraminifera from the Middle East and its distribution in the Tethys, a
} review

\begin{abstract}
One hundred and nineteen hyaline benthic foraminiferal species and subspecies belonging to fifty seven genera are originally erected from the Maastrichtian and early Paleogene rocks of six countries in the Middle East which include countries like Egypt, Jordan, Saudi Arabia, Qatar, United Arab Emirates (UAE) and Pakistan. Some of these species are also found in other places like Libya, Tunisia, Nigeria, France, Spain, Arabian Sea, Atlantic Ocean. The present study deals with paleontology, stratigraphy, paleogeography and paleoecology of these taxa in the Middle East and other Tethyan Localities.
\end{abstract}

Keywords: paleogene, calcareous benthic foraminifera, Middle East, Tethys
Volume 7 Issue 6 - 2019

\section{Haidar Salim Anan}

Emeritus Professor of stratigraphy and micropaleontology, AlAzhar University, Palestine

Correspondence: Haidar Salim Anan, Emeritus Professor of stratigraphy and micropaleontology, Al-Azhar University, Gaza P. O. Box I | 26, Palestine, Email profana@gmail.com

Received: July 16, 2019 | Published: November II, 2019

\section{Introduction}

The present paper aims to highlight the paleontology, stratigraphy paleogeography, paleoecology and biogeography of the 119 Maastrichtian-early Paleogene calcareous benthic foraminiferal species and subspecies which were originally erected by many authors from six Middle East countries (Maps 1 \& 2): (1) Egypt: Nakkady, ${ }^{1-3}$ LeRoy, ${ }^{4}$ Said \& Kenawy, ${ }^{5}$ Abdou \& Abdel Kireem, ${ }^{6}$ Anan, ${ }^{7-27}$ Aly et al., ${ }^{28}$ (2) Jordan: Futyan, ${ }^{29}$ (3) Saudi Arabia: Hasson ${ }^{30}$ (4) Qatar: Hewaidy \& Al-Hitmi ${ }^{31}$ (5) UAE: Anan ${ }^{32-37}$ and (6) Pakistan: Haque, ${ }^{38-40}$ Nomura \& Brohi, ${ }^{41}$ and many studies are recorded some of these taxa in a wider geographic areas in the Tethys, from Atlantic to Indian Oceans via Mediterranean (Map 3).

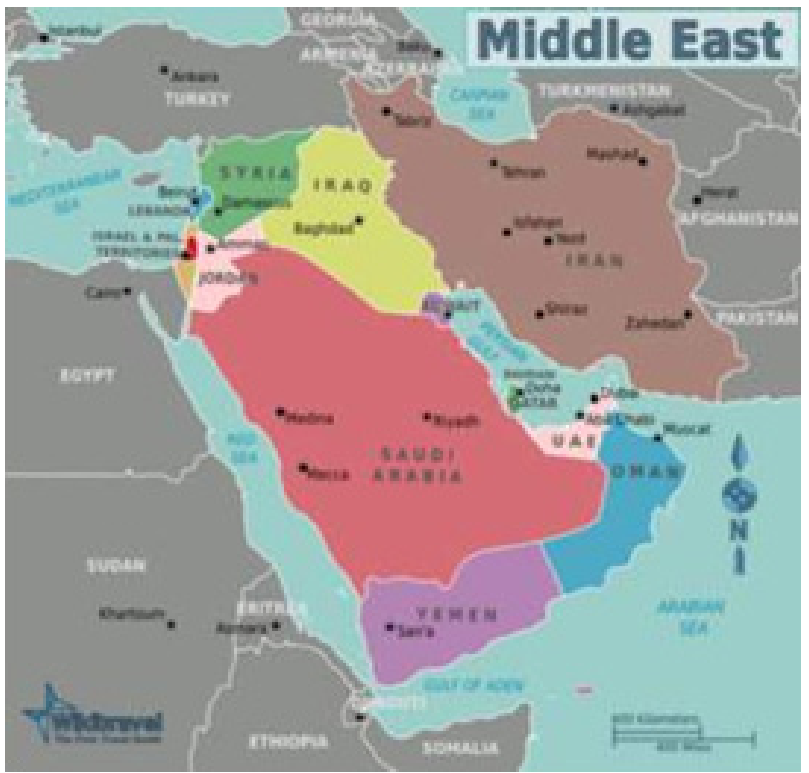

Map I Map of the six Middle East countries, from west to east: Egypt, Jordan, Saudi Arabia, Qatar, United Arab Emirates (UAE) and Pakistan.

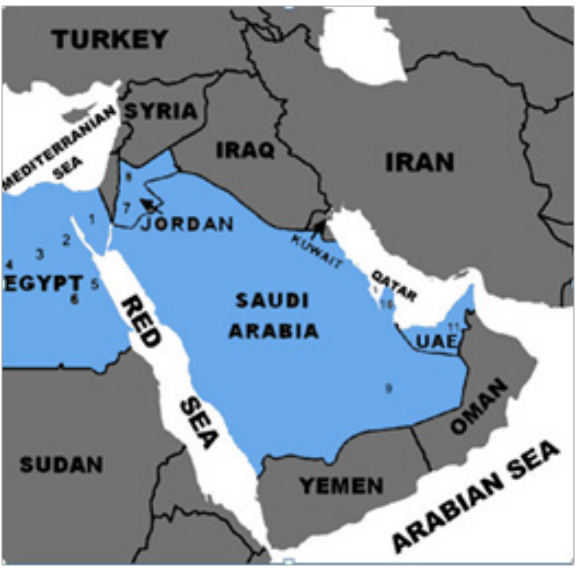

Map 2 Map of the five Arab Middle East countries with the locations of some diagnostic studied sections, which some species were erected: Egypt (I. Sinai, 2. El Sheikh Fadl, Mellaha, Wadi EdDakhl sections, 3. Fayoum, 4. Maqfi section (Farafra area), 5. Duwi section, 6. Luxor section), Jordan (7.Tell Burma section, 8. Wadi Arab section), Saudi Arabia (9. Rub' al Khali Basin), Qatar (I0 Dukhan oil field), United Arab Emirates, UAE (I I. Jabal Hafit, J. Malaqet).

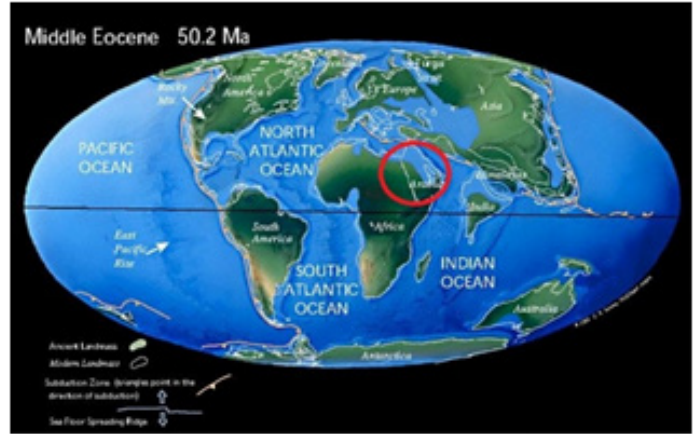

Map 3 Paleogeographic map of the Early Paleogene (particularly Middle Eocene) including the location of the Middle East (Egypt and Arabia), after. 


\section{Systematic paleontology}

The taxonomy of Loeblich \& Tappan ${ }^{42}$ is followed here. One hundred and nineteen of the erected Maastrichtian and/or Paleogene small benthic foraminiferal species and subspecies from six Middle East countries are illustrated in the Plates (1-3). The stratigraphic value of it is important in the paleobiogeographic correlations in the these countries and also some other Tethyan localities.

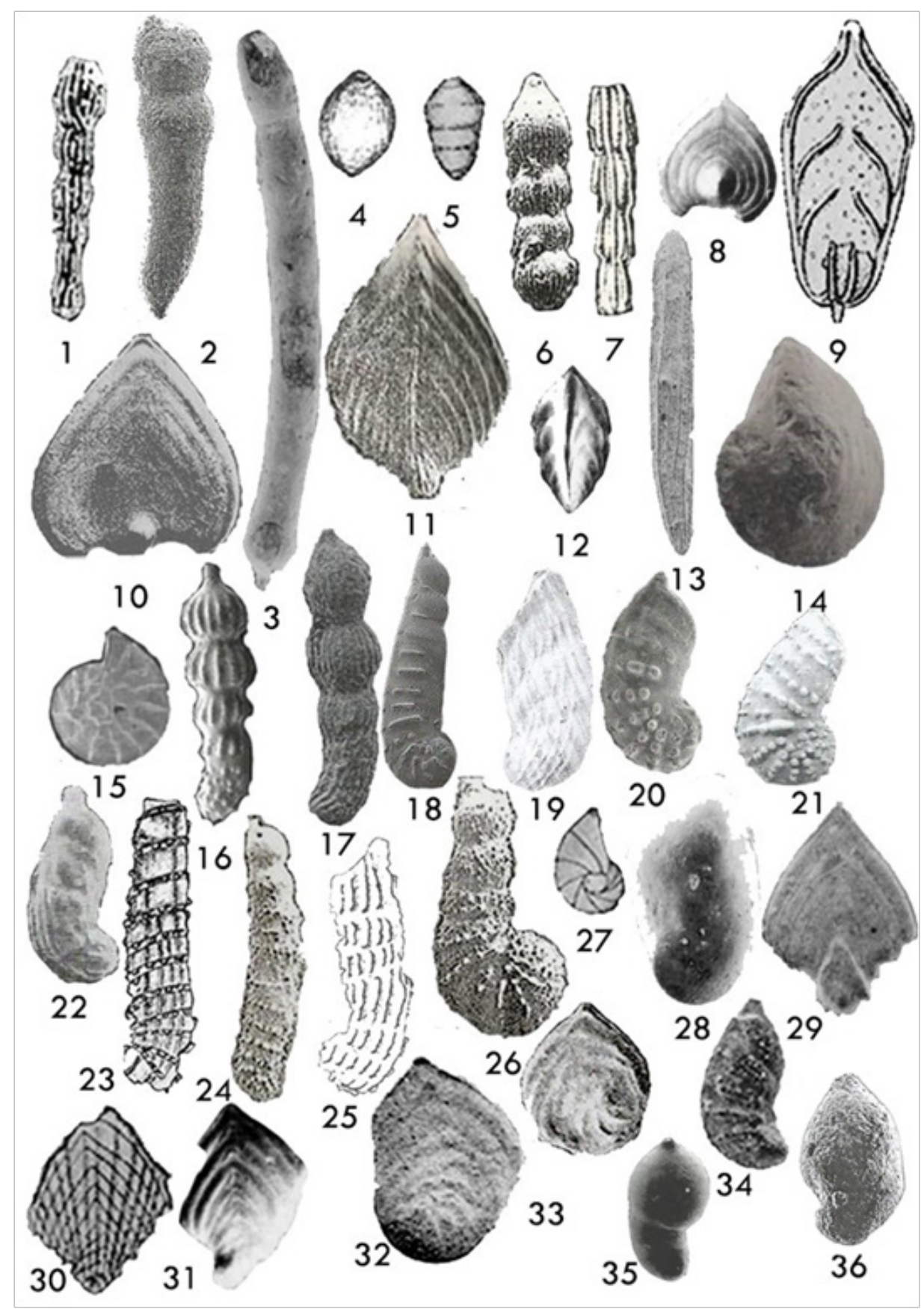

Plate I Fig. I) Dentalina Tappanae (Said \& Kenawy, 1956) × 35, 2) Laevidentalina hudae Anan, $2015 \times 70$, 3) L. salimi Anan, $2009 \times 50,4)$ Pseudonodosaria bulla (Said \& Kenawy, 1956) $\times 35$, 5) P. synaensis (Ansary, 1954) x 50, 6) Pyramidulina robinsoni (Futyan, 1976) x75, 7) P. semispinosa (LeRoy, 1953) $\times 40$, 8) Frondicularia bignoti Anan, 2002 × 50, 9) F. esnehensis Nakkady, 1950 x60, 1 0) F. nakkadyi Futyan, I $976 \times 25$, I I) Frondicularia pickeringi Futyan, I $976 \times 45$, I 2) Tristix aubertae Anan, 2002 x 30, 13) Amphimorphina youssefi Anan, $1994 \times$ 50, 14) Lenticulina ennakhali Anan, 2010 x 50, I5) L. trompi (Ansary, 1954) x 50, 16) Marginulinopsis hewaidyi Aly, 201 Ix40, I7) M. karimae Anan, $2009 \times$ 40, 18) Percultazonaria abunnasri Anan, $2015 \times$ 140, I9) P. alii Anan, $2015 \times 50,20)$ P. allami Anan, $2015 \times 60,2$ I) P. ameeri Anan, $2015 \times 30$, 22) P. carri (LeRoy, 1953) $\times 45$, 23) P. intercostata $($ Nakkady, 1950) $\times 40$, 24) P. jordanensis (Futyan, 1976) $\times 30$, 25) P. longiscata (Nakkady, 1950) $\times 25,26)$ P. wadiarabensis Futyan, $1976 \times 35,27)$ Saracenaria barnardi Ansary, $1954 \times 40,28$ ) S. leroyi Anan, $1994 \times 35,29)$ Palmula ansaryi Anan, $1994 \times 25,30) P$. gahannamensis (Ansary, 1954) x50, 31) P. salimi Anan, 2002 × 55, 32) P. undulata Nakkady, 1950 ×40, 33) P. woodi Nakkady, $1950 \times 25,34)$ Astacolus bifurcatus LeRoy, $1953 \times 40$, 35) Hemirobulina bassiounii Anan, 1994 × 50, 36) H. olae Anan, $2015 \times 40$. 


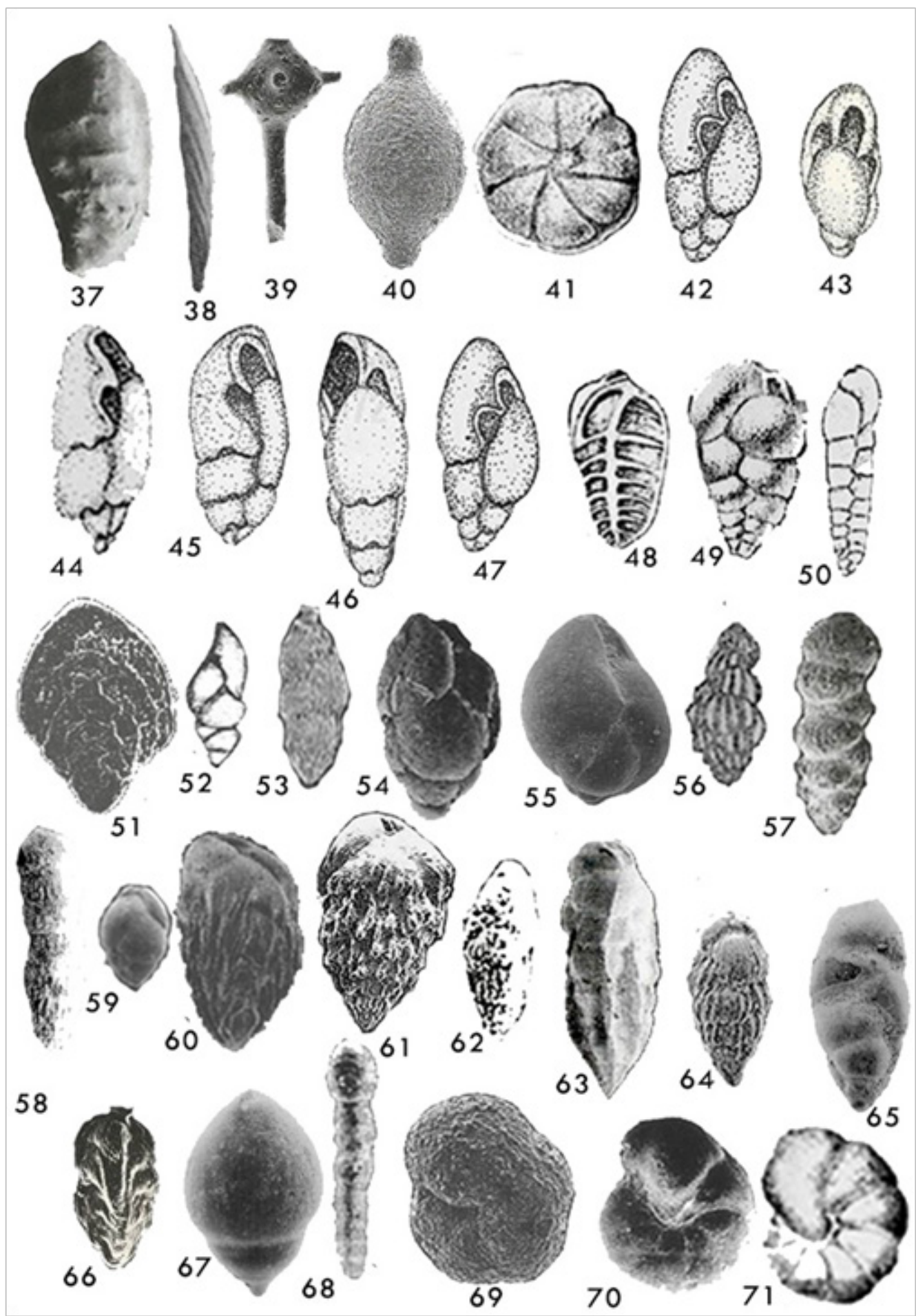

Plate 2 Fig. 37) Vaginulinopsis boukharyi Anan, $2010 \times 45$, 38) Citharina plummerae Anan, $2001 \times 50$ 39) Ramulina elkhoudaryi Anan, 2002 × 30, 40) R. futyani Anan, $2015 \times 70,41$ ) Höglundina esnaensis (LeRoy, 1953) × 50, 42) Altistoma aegyptiaca Abdou \&Abdel Kireem, $1975 \times 35,43$ ) A. compressa Abdou \& Abdel Kireem, 1975 $\times 30,44)$ A. eocaenica Abdou \& Abdel Kireem, 1975 × 30, 45) A. fayoumensis Abdou \& Abdel Kireem, $1975 \times 35,46)$ A. megaapertura Abdou \& Abdel Kireem, 1975 x 35, 47) A. rayana Abdou \& Abdel Kireem, 1975 × 35,48) Bolivina esnehensis Nakkady, 1950 × 25, 49) B. daniana Nakkady \& Talaat, 1959 x 200, 50) B. woodi Nakkady, 1950 × 75, 51) Aragonia semireticulata (LeRoy, 1953) x 75, 52) Eouvigerina hofkeri Said and Kenawy, I 956 × II 0, 53) E. maqfiensis (LeRoy, 1953) $\times 75,54)$ Neobulimina farafraensis LeRoy, 1953 × 75, 55) Turrilina hassani Anan, 2010 × 50, 56) Hopkinsina aegyptiaca Ansary, 1954 × 50, 57) Orthokarstenia higazyi (Nakkady, 1955) $\times 50$, 58) O. nakkadyi Anan, $2009 \times 50$, 59) Bulimina esnaensis LeRoy, $1953 \times 60$, 60) B. farafraensis LeRoy, $1953 \times 80,61$ ) B. leroyi Futyan, 1976 x I30, 62) B. misrensis Ansary, $1954 \times 90$, 63) B. zikoi Helal, $2002 \times 150,64)$ Uvigerina compressa Ansary, $1954 \times 70,65)$ Uvigerinella nakkadyi Anan, $1994 \times 50,66)$ Trifarina esnaensis LeRoy, 1953 × 60, 67) Ellipsogladulina arafati Anan, 2009 x 40, 68) Nodosarella minuta Said \& Kenawy, 1956 x II 0, 69) Baggina eocaenica Hewaidy \& AlHitmi, 1994 × 100, 70) Valvulineria aegyptiaca LeRoy, I 953 × 50, 7I) V. critchetti LeRoy, $1953 \times 65$. 


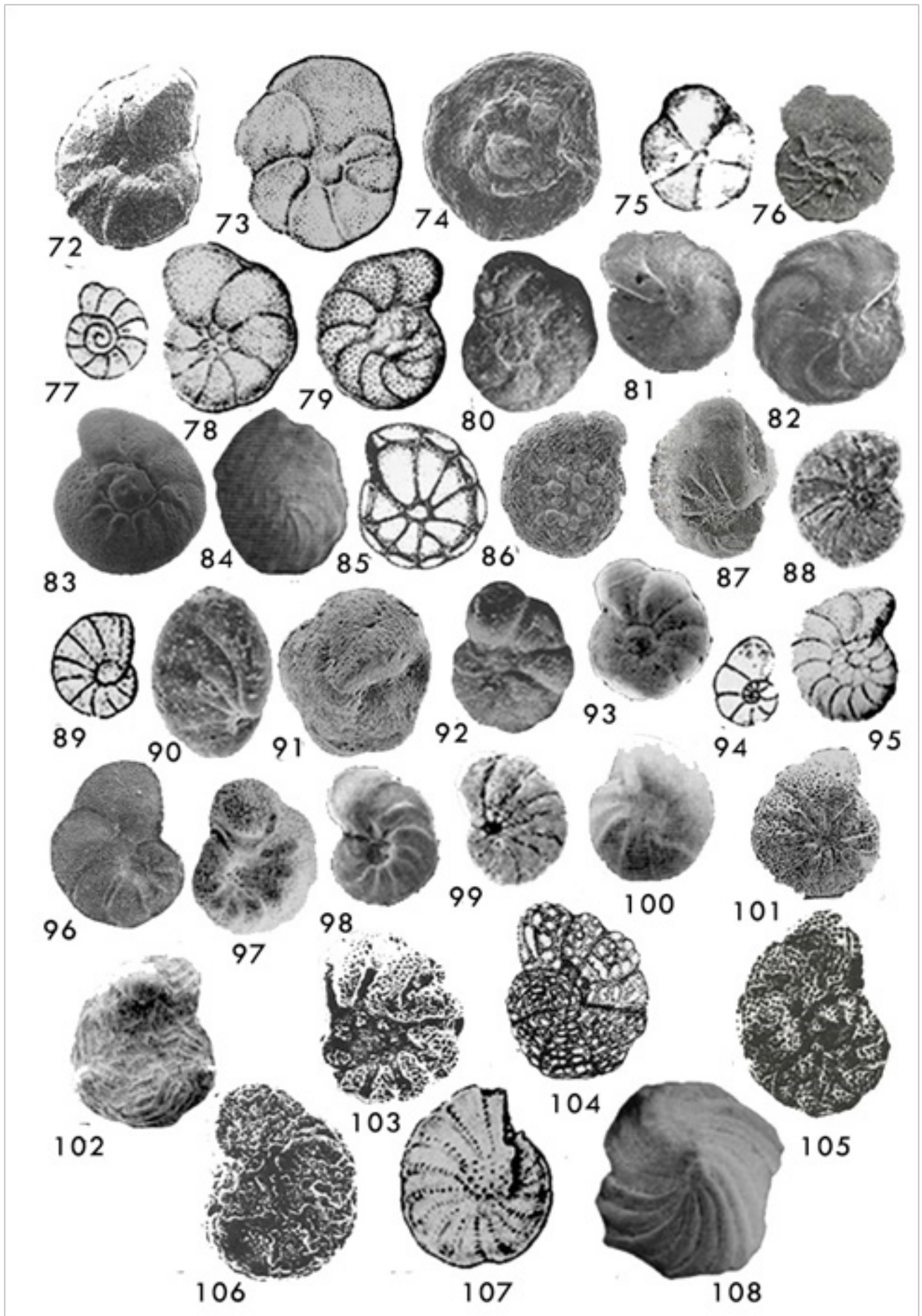

Plate 3 Fig.72)Valvulineria pseudotumeyensis Futyan, I $976 \times 75$, 73) Trochulina aegyptiaca (LeRoy, I953) x 40, 74) T. lenticularis HewaidyandAI-Hitmi, I994 xI 30, 75) Eponides mariei Said \& Kenawy, 1956 × 50, 76) Neoeponides duwi (Nakkady, 1950) x 65, 77) Cibicidoides loeblichi (Said \& Kenawy, 1956) x 45, 78) C. beadnelli (LeRoy, $1953) \times 50,79)$ C. decoratus (LeRoy, 1953) x 50, 80) C. farafraensis (LeRoy, 1953) x 50, 81) C. libycus (LeRoy, 1953) x 50, 82) C. pharaonis (LeRoy, 1953) x 50, 83) C. pseudoacutus (Nakkady, 1950) × 60, 84) Planulina berggreni Anan, 2001 × 75, 85) Epistomaria saudica Hasson, 1985, x 130, 86) Epistomaroides qatarica Hewaidy \& Al-Hitmi, 1994, x 130, 87) Nonion fayoumensis Ansary, 1954 × 60, 88) N. maadiensis Ansary, 1954 × 60, 89) N. trompi Ansary, 1954 x 60, 90$)$ Nonionella africana LeRoy, I 953 × 50, 91) Alabamina cavelieri Hewaidy \& Al-Hitmi, I $994 \times$ 150, 92) Osangularia desertorum (LeRoy, 1953) x 50, 93) Anomalinoides aegyptiacus (LeRoy, 1953) × 50, 94) A. esnehensis (Nakkady, 1950) 60, 95) A. desertorum (LeRoy, 1953) × 55, 96) A. fayoumensis (Ansary, 1 954) x 60, 97) A. leroyi Anan, 2008 × 55, 98) A. zitteli (LeRoy, 1953) x 55, 99) Gyroidinoides aegyptiaca (Ansary, 1954) xI 50, 100) G. luterbacheri Anan, 2004 ×50, 101) Angulogavelinella bandata Futyan, I 976 × 65, 102) Ornatanomalina ennakhali Anan, 201 I x50, 103) O. inflata (Hasson, I985) x75, 104) O. ornata (Hasson, 1985) x40, 105) 0. porosa (Hasson, I 985) x70, 106) O. rugosa (Hasson, 1985) x 100, 107) Elphidiella africana (LeRoy, 1953) ×55, 108) Elphidium cherifi Anan, $2010 \times 100$. 
Order Foraminiferida Eichwald, 1830

Suborder Lagenina Delage and Hérouard, 1896

Superfamily Nodosariacea Ehrenberg, ${ }^{43}$

Family Nodosariidae Ehrenberg, ${ }^{43}$

Subfamily Nodosariinae Ehrenberg, ${ }^{43}$

Genus Dentalina Risso, ${ }^{44}$

Type species Nodosaria cuvieri d'Orbigny, ${ }^{45}$

Dentalina tappanae (Said \& Kenawy $)$ (Pl. 1, fig.1)

1956 Chrysalogonium tappanae Said \& Kenawy, ${ }^{5}$ p. 134, pl. 3, fig. 10.

2003 Chrysalogonium tappanae; Ali, ${ }^{46}$ p. 119, pl. 7, fig. 6.

2011 Dentalina tappanae; Anan, ${ }^{17}$ p. 17, pl. 1, fig. 10.

Remarks: The genus Dentalina has an elongated arcuate test with twenty longitudinal ribs. According to Anan, ${ }^{17}$ this species belongs to the Dentalina. This species was recorded in Paleocene of Sinai and southern Egypt, as well as the Maastrichtian of Wadi Ed Dakhl section, west Gulf of Suez, Egypt.

Genus Laevidentalina Loeblich \& Tappan, ${ }^{47}$

Type species Laevidentalina aphelis Loeblich \& Tappan, ${ }^{48}$

Laevidentalina hudae $\operatorname{Anan}^{37}$ (P1. 1, fig. 2)

2015b Laevidentalina hudae Anan, ${ }^{37}$ p. 65, pl. 1, fig. 1.

Remarks: The Selandian species has apiculate proloculus, more widely cylindrical, less arcuate and ended by only two globular chambers than three or more semiglobular chambers. The middle-late Eocene L. salimi $\operatorname{Anan}^{34}$ (p. 3, pl. 1, fig. 2) from Jabal Hafit (UAE) has more elongated test and less number of globular last two chambers than L. hudae. L. salimi may be evolved from the Selandian L. hudae. It was recorded from the Paleocene of J. Mundassa, UAE.

Laevidentalina salimi $\operatorname{Anan}^{34}$ (P1. 1, fig. 3)

2009a Laevidentalina salimi Anan, ${ }^{34}$ p. 3, pl. 1, fig. 2.

2011 Laevidentalina salimi; Anan, ${ }^{17}$ p. 55, pl. 1, fig. 11.

Remarks: The Eocene species has an elongated, arcuate test with almost parallel sides, proloculus rounded and apiculate, wall hyaline with smooth surface, chambers nearly cylindrical and gradually grow, sutures flush limbate in the most uniserial chambers but slightly depressed in the last chambers. It was described from J. Hafit, UAE.

Genus Pseudonodosaria Boomgaart, ${ }^{49}$

Type species Glandulina discreta Reuss, ${ }^{50} 1850$

Pseudonodosaria bulla (Said \& Kenawy ${ }^{5}$ ) (Pl. 1, fig. 4)

1956 Rectoglandulina bulla Said \& Kenawy, ${ }^{5}$ p. 134, pl. 3, fig. 13.

Remarks: Loeblich \& Tappan $^{42}$ noted that the genus Pseudonodosaria is considered as a senior synonym of genus Rectoglandulina Loeblich \& Tappan. ${ }^{49}$ This species was recorded from the Paleocene of Sinai, Egypt.

Pseudonodosaria synaensis (Ansary ${ }^{51}$ ) (P1. 1, fig. 5)

1954 Pseudoglandulina synaensis Ansary, ${ }^{51}$ p. 313, pl.1, fig.14. 2013a Pseudonodosaria synaensis; Anan, ${ }^{20}$ p. 51, pl.1, fig.1.

Remarks: The description of this Eocene species falls within the range of the genus Pseudonodosaria (Loeblich \& Tappan) ${ }^{42}$. It was described from the Eocene of Sinai. It is, so far, an endemic species in Egypt.

Genus Pyramidulina Fornasini, ${ }^{52}$

Type species Pyramidulina eptagona Fornasini, ${ }^{52}$

Pyramidulina robinsoni $\left(\right.$ Futyan $^{29}$ ) (Pl. 1, fig. 6)

1976 Nodosaria robinsoni Futyan, ${ }^{29}$ p. 525, pl. 82, figs. 5, 6.

Remarks: Loeblich \& Tappan ${ }^{42}$ noted that the genus Pyramidulina Fornasini has distinct longitudinal costae, with unornamented surface in the genus Nodosaria Lamarck. This Paleocene species has uniserial test with inflated chambers covered by 22-24 closed spaced fine longitudinal ribs, which become obsolete on the upper half of the last chamber. P. robinsoni differs from $N$. limbata d'Orbigny in having fine and distinct longitudinal ribs. It was described from Middle Paleocene of Tell Burma section, S. Jordan.

\section{Pyramidulina semispinosa (LeRoy ${ }^{4}$ (Pl. 1, fig. 7)}

1953 Nodosaria semispinosa LeRoy, ${ }^{4}$ p. 41, pl. 4, fig. 10.

2001 Pyramidulina semispinosa; Hewaidy \& Strougo, ${ }^{53}$ p. 15, pl. 1, fig. 19.

2008 Pyramidulina semispinosa; Anan, ${ }^{13}$ p. 362.

Remarks: The early Eocene $P$. semispinosa was recorded only from Egypt.

Subfamily Frondiculariinae Reuss, ${ }^{54}$

Genus Frondicularia Defrance, ${ }^{55}$

Type species Renulina complanata Defrance, ${ }^{56}$

Frondicularia bignoti Anan ${ }^{10}$ (P1. 1, fig. 8)

2002a Frondicularia bignoti Anan, ${ }^{10}$ p. 632, fig. 2. 2.

2003 Palmula sp. Ali, ${ }^{45}$ pl. 6, fig. 17.

2011 Frondicularia bignoti; Anan, ${ }^{17}$ p. 55, pl. 1, fig. 12.

Remarks: This Paleocene-early Eocene species has large proloculus surrounded by four to five uniserial chevron-shaped chambers, and the first one of them completely surrounding the proloculus, smooth surface, and terminal protruding aperture. It differs from $F$. nakkadyi (Futyan) in having lesser number in chambers, which have four to five in F. bignoti than ten to thirteen in Futyan's species with its limbate flush sutures, and without two to three faint longitudinal ribs confined to the initial part of test in the latter species. It was recorded from the Paleocene of Duwi section, later from the early Eocene of southern Nile Valley sections, west Aswan, Egypt.

\section{Frondicularia esnehensis Nakkady ${ }^{1}$ (Pl. 1, fig. 9)}

1950 Frondicularia esnehensis Nakkady, ${ }^{1}$ p. 685, pl. 89, fig. 23.

2012 Frondicularia esnehensis; Ismail, ${ }^{57}$ p. 32, pl. 2, fig. 1.

Remarks: This species was recorded from the basal Esna shales (Paleocene) of Abu Durba section (south west Sinai), and Maastrichtian-Paleocene rocks of Esh El Mallaha section, west Gulf of Suez. It is, so far, an endemic to Egypt. 
Frondicularia nakkadyi Futyan ${ }^{29}$ (P1. 1, fig.10)

1976 Frondicularia nakkadyi Futyan, ${ }^{29}$ p. 528, pl. 82, fig.1.

1985 Frondicularia nakkadyi; Luger, ${ }^{58}$ p. 80, pl.4, fig. 3 .

2002a Frondicularia nakkadyi; Anan, ${ }^{10}$ p. 633, fig. 2.4.

2012 Frondicularia nakkadyi; Youssef \& Taha, ${ }^{59}$ pl. 3, fig.10.

Remarks: This species is distinguished by its large, flat, palmate test with smooth surface, but differs from the Paleocene $F$. phosphatica Russo in its lacking the central raised ridge which extends from proloculus to the aperture. It was described from Late Paleocene of Jordan, and from Maastrichtian-early Eocene of Egypt.

Frondicularia pickeringi Futyan ${ }^{29}$ (P1. 1, fig.11)

1976 Frondicularia pickeringi Futyan, ${ }^{29}$ p. 526, pl. 82, fig. 2.

Remarks: The Paleocene species has large, much compressed test distinctly rhomboidal in outline, periphery truncate in upper half of test, irregular with thin scattered transparent keel in lower half. Initial portion ornamented by two to five fine ribs which in some specimens extends towards the aperture. It was described from the late Paleocene at Tell Burma, S. Jordan.

Genus Tristix Mac Fayden, ${ }^{60}$

Type species Rabdogonium liasinum, Berthelin ${ }^{61}$

Tristix aubertae $\operatorname{Anan}^{10}$ (Pl. 1, fig.12)

2002a Tristix auberti Anan, ${ }^{10}$ p. 634, fig. 2. 6.

2011 Tristix aubertae; Anan, ${ }^{17}$ p. 55, pl. 1, fig. 13.

Remarks: This species has triangular test face, flattened, concave triangular chambers and acute periphery. It was originally described from the Danian of Duwi section, Egypt. The Tunisian form is identical with Egyptian form.

Subfamily Plectofrondiculariinae Cushman, ${ }^{62}$

Genus Amphimorphina Neugeboren, ${ }^{63}$

Type species Amphimorphina haueriana Neugeboren, ${ }^{63}$

Amphimorphina youssefi $\operatorname{Anan}^{7}$ (P1. 1, fig.13)

1994 Amphimorphina youssefi Anan, ${ }^{7}$ p. 220, fig. 8. 7.

2011 Amphimorphina youssefi; Anan, ${ }^{17}$ p. 56, pl. 2, fig. 14.

Remarks: The species has an elongate test, early portion frondicularian but nodosarian and semi-circular in the later part, sutures slightly arched in early part, but straight and horizontal at later part, periphery sharply keeled with three keels on either sides running at the whole length of the test, two supplementary raised costae are recognized in the early part on either side, aperture terminal and rounded. A. youssefi was described from the middle Eocene of Fayoum (Egypt), and later from the late Eocene of J. Malaqet (UAE).

Family Vaginulindae Reuss, ${ }^{64}$

Subfamily Lenticulininae Chapman, Parr \& Collins, ${ }^{65} 1934$

Genus Lenticulina Lamarck, ${ }^{66}$

Type species Lenticulina rotulatus Lamarck, ${ }^{66}$

Lenticulina ennakhali Anan ${ }^{36}$ (Pl. 1, fig.14) 2010c Lenticulina ennakhali Anan, ${ }^{36}$ p. 20, fig. 2.

2011 Lenticulina ennakhali; Anan, ${ }^{17}$ p. 56, pl. 2, fig. 14.

Remarks: The most outstanding characteristics of the Paleoceneearly Eocene $L$. ennakhali when compared to other species of Lenticulina are the tapering last chamber in an elongate test $(1 / \mathrm{b}=1.33)$, flush sutures and sharp periphery with faint keel. L. ennakhali was described from Dakhla Shale of Abu Zenima section, Sinai, Egypt.

Lenticulina trompi (Ansary ${ }^{51}$ ) (Pl. 1, fig.15)

1954 Robulus trompi Ansary, ${ }^{51}$ p. 314, pl. 1, fig. 2.

1992 Lenticulina trompi; Hussein, ${ }^{67}$ p. 110, fig. 2. 6.

2000 Lenticulina trompi; Abul-Nasr, ${ }^{68}$ p. 72, fig. 15. 1, 2.

2011 Lenticulina trompi; Aly et al., ${ }^{28}$ p. 90, pl. 2, fig. 8 .

2013a Lenticulina trompi; Anan, ${ }^{20}$ p. 51, pl. 1, figs. 2-4.

Remarks: This Eocene species was described from Sinai, Fayoum, Nile Valley (Egypt), as well as from Jordan. Abul-Nasr (2000) noted that his Lenticulina-Marginulina lineage begins with Lenticulina trompi.

Genus Marginulinopsis Silvestri, ${ }^{69} 1904$

Type species Cristellaria bradyi Goës, ${ }^{70} 1894$

Marginulinopsis hewaidyi $\mathrm{Aly}^{28}$ (Pl. 1, fig.16)

2011 Marginulinopsis hewaidyi Aly et al., ${ }^{28}$ p. 92, pl. 3, fig. 2.

Remarks: This species differs from $M$. infracompressa (Thalman) in having a lot of spines on the last chamber, and long neck ornamented by spines as well.

M. karimae $\mathrm{Anan}^{34}$ differs than M. hewaidyi in having more number of uniserial chambers, by its more longitudinal costal number extended continuously over the suture lines than independent costae on each chamber, and without faint nodes in the inter-costal space. It was recorded from the late Middle Eocene of Beni Suef, Nile Valley, Egypt.

Marginulinopsis karimae Anan $^{34}$ (P1. 1, fig.17)

2009a Marginulinopsis karimae Anan, ${ }^{34}$ p. 6, pl. 1, fig. 8 .

Remarks: The genus Marginulinopsis has numerous longitudinal costate surfaces, not nodes and beadlike tubercles surface in Percultazonaria. This species was recorded from late Eocene of J. Hafit, UAE.

Genus Percultazonaria Loeblich \&Tappan, ${ }^{46}$

Type species Cristellaria subaculeata Cushman, ${ }^{71}$

Percultazonaria abunnasri $\operatorname{Anan}^{23}$ (Pl. 1, fig.18)

2015a Percultazonaria abunnasri, Anan, ${ }^{23}$ p. 16, pl. 1, fig. 1.

Remarks: According to Loeblich \& Tappan ${ }^{42}$ the type species of the genus Percultazonaria was recorded from Holocene time, but it now ranges from Cretaceous to Holocene. According to Abul$\mathrm{Nasr}^{68}$ (figs. 9, 10) the two middle Eocene species: P. abunnasri and $P$. benisuefensis (sensu Hussein ${ }^{72}$ ) most probably have been evolved from two different stocks, which have progressive of raised costate ridges, in one hand: Lenticulina trompi-Marginulinopsis $\mathrm{cf}$. fragaria-Marginulina benisuefensis (sensu Abul-Nasr ${ }^{68}$ ) lineage, 
and raised nodes in another: L. chitanii-M. brantlyi-M. waiparaensis lineage, and these two lineages have been ended by the late Eocene Marginulina costata. It was recorded from Darat Formation of Wadi Tayiba section, Sinai, Egypt.

Percultazonaria alii $\operatorname{Anan}^{23}$ (Pl. 1, fig.19)

2015a Percultazonaria alii Anan, ${ }^{23}$ p. 16, pl. 1, fig. 2.

Remarks: This species differs from early Eocene P. longiscata of Nakkady by its interrupted ridges at the sutures. The more breadth test and raised ridges running across the sutures of the Sweden Paleocene Astacolus paleocenicus Brotzen ${ }^{73}$ (1948) which often interrupted over the sutures, make it differs from the $P$. alii. It was recorded from Kurkur Naqb Dungul section, west Aswan, Egypt.

Percultazonaria allami $\operatorname{Anan}^{23}$ (P1. 1, fig. 20)

2015a Percultazonaria allami Anan, ${ }^{23}$ p. 17, pl. 1, fig. 3.

Remarks: This Paleocene-middle Eocene species has inner margin slightly curved, while the outer margin curved with keel. It was recorded from Beni Suef Formation of Warshet ErRukham section, Nile Valley, Egypt.

\section{Percultazonaria ameeri $\operatorname{Anan}^{23}$ (Pl. 1, fig. 21)}

2015a Percultazonaria ameeri Anan, ${ }^{23}$ p. 17, pl. 1, fig. 4.

Remarks: This Paleocene species can be recognized by its large size test, and conspicuous sutural nodes along the coiled and inclined uniserial portions of the test. It was recorded from the Paleocene of the Maqfi section, Egypt.

\section{Percultazonaria carri (LeRoy ${ }^{4}$ ) (Pl. 1, fig. 22)}

1953 Marginulina carri LeRoy, ${ }^{4}$ p. 38, pl. 4, figs. 4, 5.

2001 Marginulina carri; Hewaidy \& Strougo, ${ }^{52}$ p. 17, pl. 2, fig. 9. 2015a Percultazonaria carri; Anan, ${ }^{23}$ p. 20, pl. 1, fig. 8.

Remarks: This early Eocene species was recorded only in the central Egypt. It seems to be confined, so far, in Egypt.

\section{Percultazonaria intercostata (Nakkady ${ }^{1}$ ) (Pl. 1, fig. 23)}

1950 Marginulina wetherilli Jones var. intercostata Nakkady, ${ }^{1} \mathrm{p}$. 684, pl. 89, fig. 12.

1996 Marginulina wetherilli intercostata; Aref \& Youssef, ${ }^{74}$ p. 553 , pl. 3 , figs. 4,5 .

2015a Percultazonaria intercostata; Anan, ${ }^{23}$ p.21, pl. 1, fig. 10.

Remarks: This species was recorded from the Paleocene-Eocene in many parts in Egypt: Sinai, Luxor and Farafra area of Egypt. It seems that this species has mainly common feature with $P$. tuberculata (Plummer ${ }^{75}$ ) which make some confusion by some authors between these two species (tuberculata and intercostata). P. intercostata has spite fine parallel ridges beside its beaded tuberculate sutures, not only beaded tuberculate in Plummer's species.

\section{Percultazonaria jordanensis (Futyan ${ }^{29}$ ) (P1. 1, fig. 24)}

1976 Marginulina jordanensis, Futyan, ${ }^{29}$ p. 525, pl. 81, figs. 5, 6.

2015a Percultazonaria jordanensis; Anan, ${ }^{23}$ p. 21, pl. 1, fig. 11.

Remarks: This Paleocene species resembles the Eocene $P$. wetherelli Jones in its more compressed with larger initial coiled portion, less inflated chambers, lacks the last two inflated chambers and depressed sutures. P. jordanensis was described from Tell Burma and Wadi Arab sections, Jordan.

\section{Percultazonaria longiscata (Nakkady ${ }^{1}$ ) (P1. 1, fig. 25)}

1950 Marginulina wetherilli Jones var. longiscata Nakkady, ${ }^{1} \mathrm{p}$. 684 , pl. 89 , fig. 13

2010b Marginulina longiscata; Anan, ${ }^{16}$ p. 41, fig. 2.

2015a Percultazonaria longiscata; Anan, ${ }^{23}$ p. 6, pl. 2, fig. 12.

Remarks: This Eocene species has elongated ridges, but interrupted at sutures, which are not raised or tubercled. It was recorded from Luxor, Farafra area and Duwi section, Egypt.

\section{Percultazonaria wadiarabensis Futyan ${ }^{29}$ (Pl. 1, fig. 26)}

1976 Vaginulinopsis wadiarabensis Futyan, ${ }^{29}$ p. 524, pl. 81, figs. 7-9.

2015a Percultazonaria wadiarabensis; Anan, ${ }^{23}$ p. 24, pl. 2, fig. 15.

Remarks: The genus Percultazonaria differs from Vaginulinopsis in more prominent early coil, and in its elevated, costate, nodose sutural ornamentation. This Paleocene species resembles $P$. tuberculata (Plummer ${ }^{75}$ ), but has a larger coiled initial portion, stouter and flanged test, spinose periphery and surface, 1-2 inflated uniserial chambers instead of 4-6 laterally compressed chambers in Plummer's species which are longer than high. It was recorded from Jordan and Egypt.

Genus Saracenaria Defrance, ${ }^{56}$

Type species Saracenaria italica Defrance, ${ }^{56}$

Saracenaria barnardi Ansary ${ }^{51}$ (Pl. 1, fig. 27)

1954 Saracenaria barnardi Ansary, ${ }^{51}$ p. 315, pl. 2, fig. 3.

2013a Saracenaria barnardi; Anan, ${ }^{20}$ p. 51, pl. 1, figs. 5-6.

Remarks: It was described from the Eocene of Fayoum area, Egypt.

Saracenaria leroyi Anan, ${ }^{7}$ (P1. 1, fig. 28)

1994 Saracenaria leroyi Anan, ${ }^{7}$ p. 222, fig. 8. 14, 15.

2009a Saracenaria leroyi; Anan, ${ }^{34}$ p. 7, pl. 1, fig. 10.

Remarks: It was described from the middle Eocene of Fayoum and Sinai of Egypt, and later from the Eocene of J. Hafit, UAE.

Subfamily Palmulinae Saidova, ${ }^{76}$

Genus Palmula Lea, ${ }^{77}$

Type species Palmula sagittaria Lea, ${ }^{77}$

Palmula ansaryi $\operatorname{Anan}^{7}$ (P1. 1, fig. 29)

1994 Palmula ansaryi Anan, ${ }^{7}$ p. 222, fig. 8. 14, 15.

1998 Palmula ansaryi; Hussein, ${ }^{72}$ p. 216, fig. 2 a.

2002 Palmula ansaryi; Helal, ${ }^{78}$ p. 114, pl. 2, fig. 6.

2011 Palmula ansaryi; Aly et al., ${ }^{28}$, p. 94, pl. 3, fig. 8.

2013a Palmula ansaryi; Anan, ${ }^{20}$ p. 51, pl. 1, fig. 7.

Remarks: Eocene species was described from the middle Eocene in many sites from Egypt

Palmula gahannamensis (Ansary ${ }^{51}$ ) (P1. 1, fig. 30) 
1954 Flabellina gahannamensis Ansary, ${ }^{51}$ p. 313, pl.1, fig.1.

1998 Palmula gahannamensis; Hussein, ${ }^{72}$ p. 216.

2013a Palmula gahannamensis; Anan, ${ }^{20}$ p. 51, pl. 1, fig. 8 .

Remarks: The Eocene P. gahannamensis was recorded in the Mokattam area and Warshet ErRukham, Beni Suef, Egypt.

Palmula salimi Anan ${ }^{10}$ (Pl. 1, fig. 31)

2002a Palmula salimi Anan, ${ }^{10}$ p. 638, fig. 2. 7.

2011 Palmula salimi; Anan, ${ }^{17}$ p. 59, pl. 2, fig. 20.

Remarks: Paleocene species was recorded from Duwi section. It is an excellent marker Paleocene species in Egypt.

Palmula undulata Nakkady ${ }^{1}$ (P1. 1, fig. 32)

1950 Palmula woodi var. undulata Nakkady, ${ }^{1}$ p. 685, pl. 89, fig. 25.

1986 Palmula woodi undulata; Anan \& Hewaidy ${ }^{79}$ p. 22, pl. 2, fig. 9.

2009 b Palmula woodi undulata; Anan, ${ }^{14}$ p. 35 , pl. 1, fig. 3.

Remarks: Nakkady (1950) recorded this subspecies from the Paleocene of Duwi section, and later from central Egypt.

Palmula woodi Nakkady ${ }^{1}$ (Pl. 1, fig. 33)

1950 Palmula woodi Nakkady, ${ }^{1}$ p. 684, pl. 89, fig. 24.

1976 Palmula woodi; Aubert \& Berggren, ${ }^{80}$ p. 417, pl. 3, fig. 12.

1985 Palmula woodi; Luger, ${ }^{58}$ p. 84, pl. 5, fig. 9.

1988 Palmula w. woodi; Anan \& Sharabi, ${ }^{81}$ p. 207, pl.1, fig. 26.

2003 Palmula woodi; Ali, ${ }^{45}$ pl. 6, figs. 12,13.

2009b Palmula w. woodi; Anan, ${ }^{14}$ p. 35, pl. 1, fig. 4.

Remarks: It was recorded from the Paleocene of Duwi section and has wide geographic distribution in Egypt, Jordan and Tunisia.

Subfamily Marginulininae Wedekind, ${ }^{82}$

Genus Astacolus de Montfort, ${ }^{83}$

Type species Astacolus crepidulatus de Montfort, ${ }^{83}$

Astacolus bifurcatus LeRoy ${ }^{4}$ (Pl. 1, fig. 34)

1953 Astacolus bifurcatus LeRoy, ${ }^{4}$ p.19, pl. 4, figs.1, 2.

1993b Astacolus bifurcatus; Anan, ${ }^{84}$ p. 658, pl. 2, fig.16.

2008 Astacolus bifurcatus; Anan, ${ }^{13}$ p. 363, pl. 1, fig.3.

Remarks: This Maastrichtian-Paleocene species was recorded from many parts in Egypt and UAE.

Genus Hemirobulina Stache, ${ }^{85} 1864$

Type species Cristellaria (Hemirobulina) arcuatula Stache, ${ }^{85}$

\section{Hemirobulina bassiounii Anan? (PI. 1, fig. 35)}

1994 Hemirobulina bassiounii Anan, ${ }^{7}$ p. 223, fig. 8.16.

2017 Hemirobulina bassiounii; Hewaidy et al., ${ }^{86}$ p. 85, pl. 3, fig. 30.
Remarks: This Eocene species was described from late middle Eocene of Egypt and J. Hafit, UAE.

Hemirobulina olae $\operatorname{Anan}^{37}$ (P1.1, fig. 36)

2015b Hemirobulina olae Anan, ${ }^{37}$ p. 71 , pl. 1, fig. 8.

Remarks: Selandian species differs from the Paleocene-Early Eocene H. hamuloides (Brotzen) by its more inflated test, circular in cross section and rounded periphery. It was described by J. Mundassa, UAE.

Subfamily Marginulininae Wedekind, ${ }^{82}$

Genus Vaginulinopsis Silvestri, ${ }^{87}$

Type species Vaginulina solute Silvestri var. carinata Silvestri, ${ }^{88}$

Vaginulinopsis boukharyi Anan ${ }^{16}$ (Pl. 2, fig. 37)

2010b Vaginulinopsis boukharyi Anan, ${ }^{16}$ p. 30, pl. 1, fig. 12.

2011 Vaginulinopsis boukharyi; Anan, ${ }^{17}$ p. 59, pl. 2, fig. 22.

Remarks: This species has a large test, small involute planispiral stage, gradually increase 9-10 chambers in the uniserial later stage with horizontal raised sutures and dorsal end aperture. It was recorded from the Paleocene of J. Duwi.

Subfamily Vaginulininae Reuss, ${ }^{64}$

Genus Citharina d'Orbigny,

Type species Vaginulina (Citharina) strigillata Reuss, ${ }^{64}$

Citharina plummerae Anan' (Pl. 2, fig. 38)

2001 Citharina plummerae Anan, ${ }^{9}$ p. 135, pl. 1, fig. 1.

2017 b Citharina plummerae; Anan, ${ }^{25}$ p. 280, fig. 6.17a.

2017 Citharina plummerae; Hewaidy et al., ${ }^{86}$ p. 85, pl. 3, fig. 22.

Remarks: It has wing-shaped flattened test with thin acuminates, globular to ovate proloculus, gradually increasing later uniserial chambers in breadth, numerous regular spaced longitudinal striae parallel to direction of growth, raised oblique curved sutures, protruding aperture at dorsal angle. It is an excellent marker Paleocene species in Egypt.

Family Polymorphinidae d'Orbigny, ${ }^{45}$

Subfamily Ramulininae Brady, ${ }^{89}$

Genus Ramulina Jones, ${ }^{90} 1875$

Type species Ramulina laevis Jones, ${ }^{90}$

Ramulina elkhoudaryi $\operatorname{Anan}^{11}$ (Pl. 2, fig. 39)

2002b Ramulina elkhoudaryi Anan, ${ }^{11}$ p. 143, fig. 2. 2.

2014a Ramulina elkhoudaryi; Anan, ${ }^{21}$ p. 66, pl. 1, figs. 3, 4.

Remarks: The Paleocene figured specimen $R$. navarroana of Youssef \& $\operatorname{Taha}^{59}$ (p. 15, pl. 4, fig. 19) is closely related to Anan's species $R$. elkhoudaryi. For that, the stratigraphic range of $R$. elkhoudaryi is extended from the Maastrichtian-Paleocene of Egypt.

Ramulina futyani $\operatorname{Anan}^{37}$ (Pl. 2, fig. 40)

2015b Ramulina futyani Anan, ${ }^{37}$ p. 72, pl. 1, fig. 11.

Remarks: Paleocene species differs from $R$. pseudoaculeata 
(Olsson) by its more globular and hispid test. It likes $P$. globosa (Montagu) in its globular test but differs in its hispid surface. It was recorded from the Selandian of J. Mundassa, UAE.

Suborder Robertinina Loeblich \& Tappan, ${ }^{91}$

Superfamily Ceratobuliminacea Cushman, ${ }^{62}$

Family Epistominidae Wedekind, ${ }^{82}$

Subfamily Epistomininae Wedekind, ${ }^{82}$

Genus Höglundina Brotzen, ${ }^{73}$

Type species Höglundina elegans d'Orbigny, ${ }^{92}$

Höglundina esnaensis (LeRoy ${ }^{4}$ (Pl. 2, fig. 41)

1953 Epistomina esnaensis LeRoy, ${ }^{4}$ p. 29, pl. 5, figs 7-9.

1956 Höglundina esnaensis; Said \& Kenawy, ${ }^{5}$ p. 152, pl. 6, fig. 14.

2008 Höglundina esnaensis; Anan, ${ }^{13}$ p. 363.

Remarks: Loeblich \& Tappan ${ }^{42}$ noted that the genus Höglundina differs from the genus Epistomina Terquem by its biconvex test and curved backward to the peripheral keel suture on spiral side with slightly elevation than the latter genus in its planoconvex test and limbate elevated sutures. This early Eocene species was recorded, so far, from Egypt.

Suborder Rotaliina Delage \& Hérouard, 1896

Superfamily Bolivinacea Glaessner, ${ }^{93}$

Family Bolivinidae Glaessner, ${ }^{93}$

Genus Altistoma de Klasz \& Rérat, ${ }^{94}$

Type species Altistoma scalaris de Klasz \& Rérat, ${ }^{94}$

Altistoma aegyptiaca Abdou \& Abdel Kireem ${ }^{6}$ (P1. 2, fig. 42)

1975 Altistoma aegyptiaca Abdou \& Abdel Kireem, ${ }^{6}$ p. 48, pl. 1, fig. 1.

2000 Altistoma aegyptiaca; Shahin, ${ }^{95}$ p. 201, fig.13. 1.

Remarks: This middle Eocene species was recorded, so far, from Fayoum area, and Sinai of Egypt.

\section{Altistoma compressa Abdou \& Abdel Kireem ${ }^{6}$ (Pl. 2, fig. 43)}

1975 Altistoma compressa Abdou \& Abdel Kireem, ${ }^{6}$ p. 49, pl. 1 , fig. 2.

\section{Altistoma eocaenica Abdou \& Abdel Kireem6 (Pl. 2, fig. 44)}

1975 Altistoma eocaenica Abdou \& Abdel Kireem, ${ }^{6}$ p. 50, pl. 1, fig. 6 .

2000 Altistoma eocaenica; Shahin, ${ }^{95}$ p. 201, fig.13. 2.

Altistoma fayoumensis Abdou \& Abdel Kireem6 (Pl. 2, fig. 45)

1975 Altistoma fayoumensis Abdou \& Abdel Kireem, ${ }^{6}$ p. 51, pl. 1, fig. 3 .

1994 Altistoma fayoumensis; Anan, ${ }^{7}$ p. 223.

Altistoma megaapertura Abdou \& Abdel Kireem ${ }^{6}$ (Pl. 2, fig. 46)

1975 Altistoma megaapertura Abdou \& Abdel Kireem, ${ }^{6}$ p. 51, pl. 1, fig. 4.
Altistoma rayana Abdou \& Abdel Kireem, ${ }^{6}$ (P1. 2, fig. 47)

1975 Altistoma rayana Abdou \& Abdel Kireem, ${ }^{6}$ p. 52, pl. 1, fig. 5.

Remarks: All middle Eocene Altistoma species of Abdou \& Abdel Kireem were recorded, so far, from Fayoum area, Egypt.

Genus Bolivina d'Orbigny, ${ }^{45}$

Type species Bolivina plicata d'Orbigny, ${ }^{45}$

Bolivina esnehensis Nakkady, ${ }^{1}$ (Pl. 2, fig. 48)

1950 Bolivina esnehensis Nakkady, ${ }^{1}$ p. 688, pl. 89, fig. 17.

2009 b Bolivina esnehensis; Anan, ${ }^{14}$ p. 36.

Remarks: Eocene species was recorded from the Esna Shale of Sinai, Duwi and Luxor sections, Egypt.

Bolivina daniana Nakkady \& Talaat, in $\mathbf{N a k k a d y}^{3}$ (Pl. 2, fig. 49)

1959 Bolivina daniana Nakkady, ${ }^{3}$ p. 459, pl. 6, figs. 5, 6 .

2009b Bolivina daniana; Anan, ${ }^{14}$ p. 36.

Remarks: This Paleocene species was recorded, so far, in the Um Elghanayem section, Kharga Oasis of Egypt.

Bolivina woodi Nakkady ${ }^{1}$ (Pl. 2, fig. 50)

1950 Bolivina woodi Nakkady, ${ }^{1}$ p. 687, pl. 89, fig. 15.

2009b Bolivina woodi; Anan, ${ }^{14}$ p. 36.

Remarks: This Paleocene species was recorded from Esna Shale of Abu Durba (Sinai), Duwi and Luxor sections, Egypt.

Genus Bolivinoides Cushman, ${ }^{6}$

Type species Bolivina draco Marsson, ${ }^{96}$

\section{Bolivinoides draco aegyptiacus Anan ${ }^{24}$}

2017a Bolivinoides draco aegyptiacus Anan, ${ }^{24}$ p. 3, pl. 1, fig. 1.

Remarks: The late Maastrichtian subspecies from Abu Zenima section, Sinai of Egypt differs from the other Bolivinoides draco members by its small test, in possessing only well-developed two divergent longitudinal ribs along the smooth surface test, with another third rib in the central part between them, not two longitudinal parallel medial ribs in $B . d$. draco or reticulate ornamented surface in $B . d$. dorreeni Finlay. According to $\mathrm{Anan}^{24}$ the figured specimen of $B$. draco miliaris is not related to his proposed $B$. draco lineage due to its lack of medial longitudinal ribs which characterized the three members of B. draco lineage.

Superfamily Loxostomatacea Loeblich \& Tappan, ${ }^{97}$

Family Loxostomatidae Loeblich \& Tappan, ${ }^{97}$

Genus Aragonia Finlay, ${ }^{98}$

Type species Aragonia zelandica Finlay, ${ }^{98}$

Aragonia semireticulata (LeRoy) (Pl. 2, fig. 51)

1953 Bolivina semireticulata LeRoy, ${ }^{4}$ p. 20, pl. 8, fig. 26.

1956 Bolivinoides semireticulata; Said \& Kenawy, ${ }^{5}$ p. 140, pl. 3, fig. 48.

1983 Aragonia semireticulata; Tjalsma \& Lohmann, ${ }^{99}$ p. 23 , pl. 11, fig. 1. 
2012 Bolivinoides semireticulata; Youssef \& Taha, ${ }^{59}$ pl. 4, fig. 14.

Remarks: This Eocene species belongs to the genus Aragonia as treated by Tjalsma \& Lohmann ${ }^{99}$ more than the other two genera Bolivina d'Orbigny ${ }^{45}$ or Bolivinoides Cushman ${ }^{62}$. It was recorded from Egypt, and later from Atlantic Ocean.

Family Eouvigerinidae Cushman, ${ }^{62}$

Genus Eouvigerina Cushman, ${ }^{100}$

Type species Eouvigerina americana Cushman, ${ }^{100}$

Eouvigerina hofkeri Said \& $\operatorname{Kenawy}^{5}$ (P1. 2, fig. 52)

1956 Eouvigerina hofkeri Said \& Kenawy, ${ }^{5}$ p. 141, pl. 3, fig. 50.

Remarks: It was recorded in the Paleocene of Nekhl and Giddi sections, and in Maastrichtian of El Qusaima area, Sinai, Egypt.

\section{Eouvigerina maqfiensis (LeRoy ${ }^{4}$ (P1. 2, fig. 53)}

1953 Uvigerina maqfiensis LeRoy, ${ }^{4}$ p. 52, pl. 8, fig. 2.

1988 Uvigerina maqfiensis; Keller, ${ }^{101}$ p. 161, pl. 3, figs 11, 12.

2003 Uvigerina maqfiensis; Culver, ${ }^{102}$ p. 194, fig. 11.

2011 Eouvigerina maqfiensis; Aly et al., ${ }^{28}$ p. 102, pl. 4, figs. 25.

Remarks: LeRoy ${ }^{4}$ noted that his early Eocene U. maqfiensis tending to become uniserial in the later stage with a distinct a phialine lip on the cylindrical neck and finely hispid wall. The characters of the species most probably make it belongs to the genus Eouvigerina Cushman. This species was recorded from Egypt and Tunisia.

Superfamily Turrilinacea Cushman, ${ }^{62}$

Family Turrilinidae Cushman, ${ }^{62}$

Genus Neobulimina Cushman \& Wickenden, ${ }^{103}$

Type species Neobulimina canadensis Cushman \& Wickenden, ${ }^{103}$

Neobulimina farafraensis LeRoy ${ }^{4}$ (Pl. 2, fig. 54)

1953 Neobulimina farafraensis LeRoy, ${ }^{4}$ p. 39, pl. 8, figs. 3, 4 .

1996 Stainforthia farafraensis; Speijer et al., ${ }^{104}$ p. 125, pl. 1, fig. 7.

2005 Neobulimina farafraensis; Alegret et al., ${ }^{105}$ p. 528.

2008 Neobulimina farafraensis; Anan, ${ }^{13}$ p. 363, pl. 1, fig. 4.

Remarks: The Paleocene-early Eocene species belongs to the genus Neobulimina as originally described by LeRoy, due its subglobular chambers, loop-shaped opening aperture which extending up the terminal face, without elongate fusiform test and prolocular apical spines as the genus Stainforthia Hofker. It was recorded from Egypt.

Genus Turrilina Andreae, ${ }^{106} 1884$

Type species Turrilina alsatica Andreae, ${ }^{106}$

\section{Turrilina hassani $\operatorname{Anan}^{35}$ (Pl. 2, fig. 55)}

2010a Turrilina hassani Anan, ${ }^{35}$ p. 160, pl. 1, fig. 3.

2011 Turrilina hassani; Anan, ${ }^{17}$ p. 61, pl. 2, fig. 26

Remarks: The Eocene species differs from other Turrilina spp by its opposite $\mathrm{v}$-shaped aperture at the interiomarginal of the last chamber, and the last three chambers consist about $7 / 8$ time of the whole test. It was recorded from middle-late Eocene of J. Hafit (UAE).
Superfamily Turrilinacea Cushman, ${ }^{62}$

Family Stainforthiidae Reiss, ${ }^{107}$

Genus Hopkinsina Howe \& Wallace, ${ }^{108}$

Type species Hopkinsina danvillensis Howe \& Wallace, ${ }^{108}$

Hopkinsina aegyptiaca Ansary ${ }^{51}$ (Pl. 2, fig. 56)

1954 Hopkinsina bortotara aegyptiaca Ansary, ${ }^{51}$ p. 323, pl. 1, fig. 10.

2013a Hopkinsina aegyptiaca; Anan, ${ }^{20}$ p. 52, pl. 1, figs. 9, 10.

Remarks: This Eocene species was described from Fayoum and Sinai, and later from Qattamiya (Greater Cairo), Egypt.

Superfamily Buliminacea Jones, ${ }^{90}$

Family Siphogenerenoididae Saidova, ${ }^{109}$

Subfamily Siphogenerenoidinae Saidova, ${ }^{109}$

Genus Orthokarstenia Dietrich, ${ }^{110}$

Type species Orthocerina ewaldi Karsten, ${ }^{111}$

Orthokarstenia higazyi (Nakkady²) (Pl. 2, fig. 57)

1955 Siphogenerina higazyi Nakkady, ${ }^{2}$ p. 705, text-fig. 4.

1976 Siphogenerinoides elnaggari Futyan, ${ }^{29}$ p. 527, pl. 82, figs. $3,4,10$.

1988 Orthokarstenia higazyi; Anan \& Sharabi, ${ }^{81}$ p. 212, pl. 2, figs. 8,9 .

1993a Orthokarstenia higazyi; Anan, ${ }^{112}$ p. 315.

1998 Orthokarstenia higazyi; Anan, ${ }^{8}$ p. 372, figs. 8, 9.

2003 Orthokarstenia higazyi; Ali, ${ }^{45}$ pl.7, fig. 15.

2012 Orthokarstenia higazyi; Ismail, ${ }^{57}$ p.49, pl. 4, fig. 4.

2014b Orthokarstenia higazyi; Anan, ${ }^{22}$ p. 69, pl.1, fig. 6 .

Remarks: Paleocene species is characterized by its longitudinal ribs along the test, differs from the Maastrichtian $O$. esnehensis (Nakkady) by non-ribbed smooth test. It was described from Egypt, Jordan and UAE.

Orthokarstenia nakkadyi $\operatorname{Anan}^{14}$ (Pl. 2, fig. 58)

1998 Transitional form between $O$. higazyi (Nakkady) and $O$. eleganta (Plummer); Anan, ${ }^{8}$ p. 368, fig. 3.7.

2000 Rectuvigerina clavata (Franzenau); Sztrákos, ${ }^{113}$ p. 106, pl. 13, fig. 2.

2006 Siphogenerinoides eleganta; Ortiz \& Thomas, ${ }^{114}$ p. 132, pl. 11, fig. 4.

2007 Siphogenerinoides eleganta; Alegret \& Ortiz, ${ }^{115}$ p. 441, pl. 2, fig. 43.

2009b Orthokarstenia nakkadyi; Anan, ${ }^{14}$ p. 37, pl. 1, fig. 7.

2014b Orthokarstenia nakkadyi; Anan, ${ }^{22}$ p. 69, pl. 1, figs. 7-12.

Remarks: Orthokarstenia nakkadyi Anan $^{14}$ was recorded from the middle Paleocene (Selandian, P4a Zone) of Duwi section, Egypt, but Paleocene-early Eocene of the Esna Shale of Dababiya section by Alegret \& Ortiz (2007). The Tethyan, O. nakkadyi was recorded 
around the Ypresian/Lutetian transition in France (by Sztrákos ${ }^{113}$ ) and Spain (by Ortiz \& Thomas ${ }^{15}$ ) beside Tunisia and Egypt.

Superfamily Buliminacea Jones, ${ }^{90}$

Family Buliminidae Jones, ${ }^{90}$

Genus Bulimina d'Orbigny, ${ }^{92}$

Type species Bulimina marginata d'Orbigny, ${ }^{92}$

Bulimina esnaensis LeRoy ${ }^{4}$ (Pl. 2, fig. 59)

1953 Bulimina esnaensis LeRoy, ${ }^{4}$ p. 20, pl. 6, figs 11, 12.

2001 Bulimina esnaensis; Hewaidy \& Strougo, ${ }^{52}$ p. 17, pl. 2, fig. 16.

Remarks: This Eocene species was recorded, so far, in the Farafra area of Egypt.

\section{Bulimina farafraensis LeRoy ${ }^{4}$ (Pl. 2, fig. 60)}

1953 Bulimina farafraensis LeRoy, ${ }^{4}$ p. 21, pl. 7, figs 26, 27.

1988 Bulimina farafraensis; Keller, ${ }^{101}$ p. 161, pl. 3, figs. 13, 14.

1993 Bulimina farafraensis; Boltovskoy \& Vera Ocampo, ${ }^{116} \mathrm{p}$ 147, pl. 1, fig. 16.

1994 Bulimina farafraensis; Speijer ${ }^{117}$ p. 110, pl. 1, fig. 8 .

2003 Bulimina farafraensis; Culver, ${ }^{102}$ p. 189, fig. 8; p. 194, fig. 11 .

2007 Bulimina farafraensis; Alegret \& Ortiz, ${ }^{115}$ p. 440, pl. 1, fig. 20

2012 Bulimina farafraensis; Youssef \& Taha, ${ }^{59}$ pl. 4, fig. 15.

Remarks: The Eocene species was recorded in Egypt, Tunisia, Spain and Arabian Sea.

Bulimina leroyi Futyan ${ }^{29}$ (Pl. 2, fig. 61)

1976 Bulimina leroyi Futyan, ${ }^{29}$ p. 530, pl. 82, figs. 11, 12.

Remarks: This early Eocene species has finer costae and these cover the lower third of the test.

Bulimina misrensis Ansary ${ }^{51}$ (Pl. 2, fig. 62)

1954 Bulimina jarvisi misrensis Ansary, ${ }^{51}$ p. 322, pl.1, fig. 9.

1992 Bulimina jarvisi misrensis; Cherif et al., ${ }^{118}$ p. 50, pl. 3, fig. 9

2013a Bulimina misrensis; Anan, ${ }^{20}$ p. 52, pl. 1, fig. 11.

Remarks: It was described from the late Eocene of Sinai (Egypt) and later from J. Hafit.

Bulimina zikoi Helal ${ }^{78}$ (Pl. 2, fig. 63)

2002 Bulimina zikoi Helal, ${ }^{78}$ p. 118, pl. 3, figs. 3, 4.

Remarks: This species was described from the middle-late Eocene of Fayoum area, west Egypt.

Subfamily Uvigerininae Haeckel, ${ }^{119}$

Genus Uvigerina d'Orbigny, ${ }^{92}$

Type species Uvigerina pygmaea d'Orbigny, ${ }^{92}$

Uvigerina compressa Ansary ${ }^{51}$ (Pl. 2, fig. 64)
1954 Uvigerina mediterranea compressa Ansary, ${ }^{51}$ p. 323, pl.1, fig. 15 .

1994 Uvigerina compressa; Anan, ${ }^{7}$ p. 224, fig. 9.1.

2013a Uvigerina compressa; Anan, ${ }^{20}$ p. 52, pl. 1, figs. 12, 13.

Remarks: This species was described from the Eocene rocks of Sinai, and later from Qattamiya area (Greater Cairo).

Subfamily Uvigerininae Haeckel, ${ }^{119}$

Family Uvigerinidae Haeckel, ${ }^{119}$

Subfamily Uvigerininae Haeckel, ${ }^{119}$

Genus Uvigerinella Cushman, ${ }^{100}$

Type species Uvigerina (Uvigerinella) californica Cushman, ${ }^{100}$

Uvigerinella nakkadyi $\operatorname{Anan}^{7}$ (P1. 2, fig. 65)

1994 Uvigerinella nakkadyi Anan, ${ }^{7}$ p. 224, fig. 9. 5.

2000 Uvigerinella nakkadyi; Shahin, ${ }^{95}$ p. 200, fig. 12. 11, 12.

2007b Uvigerinella nakkadyi; Anan, ${ }^{33}$ p. 73.

2011 Uvigerinella nakkadyi; Anan, ${ }^{17}$ p. 62, pl. 3, fig. 29.

Remarks: This species was originally described from the Bartonian of Fayoum, from the Late Eocene-Oligocene of Sinai, Egypt $\left(\right.$ Shahin $\left.^{95}\right)$, and later on Priabonian of J. Malaqet, UAE $\left(\operatorname{Anan}^{33}\right)$.

Subfamily Angulogerininae Galloway, ${ }^{120}$

Genus Trifarina Cushman, ${ }^{121} 1923$

Type species Trifarina bradyi Cushman, ${ }^{121}$

Trifarina esnaensis $\operatorname{LeRoy}^{4}$ (Pl.2, fig. 66)

1953 Trifarina esnaensis LeRoy, ${ }^{4}$ p. 52, pl. 8, figs. 5, 6.

1974a Trifarina esnaensis; Berggren, ${ }^{122}$ p. 438, pl. 1, fig. 6.

1985 Trifarina esnaensis; Luger, ${ }^{58}$ p. 108, pl. 7, fig. 11.

1988 Trifarina esnaensis; Keller, ${ }^{101}$ p. 161, pl. 3, figs. 15, 16.

1993b Trifarina esnaensis; Anan, ${ }^{84}$ p. 569, pl. 3, fig. 9.

2003 Trifarina esnaensis; Culver, ${ }^{102}$ p.192, fig.10.

Remarks: Paleocene-Eocene species was recorded in the most facies of Egypt, UAE, Tunisia, North Atlantic.

Superfamily Fursenkoinacea Loeblich \& Tappan, ${ }^{123}$

Family Fursenkoinidae Loeblich \& Tappan, ${ }^{123}$

Genus Fursenkoina Loeblich \& Tappan, ${ }^{123}$

Type species Virgulina squammosa d'Orbigny, ${ }^{92}$

\section{Fursenkoina dubia $\left(\right.$ Haque $^{38}$ )}

1956 Virgulina dubia Haque, ${ }^{38}$ p. 129, pl. 25, figs. 3, 4.

2019a Fursenkoina dubia; Anan, ${ }^{24}$ p. 32, fig. 9a-d.

Remarks: Paleocene-early Eocene species is characterized by its elongate biserial twisted smooth chambers throughout the test with rounded to ovate in section. It was recorded, so far, from Pakistan and Egypt.

Superfamily Pleurostomellacea Reuss, ${ }^{124}$ 
Family Pleurostomellidae Reuss, ${ }^{124}$

Subfamily Pleurostomellinae Reuss, ${ }^{124}$

Genus Ellipsoglandulina Sivestri, ${ }^{125}$

Type species Ellipsoglandulina laevigata Sivestri, ${ }^{125}$

Ellipsogladulina arafati Anan ${ }^{15}$ (P1. 2, fig. 67)

2009c Ellipsogladulina arafati Anan, ${ }^{15}$ p. 111, fig. 2.

2011 Ellipsogladulina arafati; Anan, ${ }^{17}$ p. 62, pl. 3, fig. 30.

Remarks: This early Eocene species was recorded from Abu Zenima section, Sinai, Egypt.

Genus Nodosarella Rzehak, ${ }^{126}$

Type species Lingulina tuberosa Gümbel, ${ }^{127}$

Nodosarella minuta Said \& Kenawy ${ }^{5}$ (P1. 2, fig. 68)

1956 Nodosarella minuta Said \& Kenawy, ${ }^{5}$ p. 145, pl. 4, fig. 30.

1992 Nodosarella minuta; Ismail, ${ }^{128}$ p. 238, pl. 2, fig. 5.

2011 Nodosarella minuta; Anan, ${ }^{17}$ p. 20, pl. 2, fig. 3.

Remarks: This Paleocene species was recorded from Sinai, but Maastrichtian of Sufr El Dara, Red Sea coast, Egypt

Superfamily Pleurostomellacea Reuss, ${ }^{64}$

Family Pleurostomellidae Reuss, ${ }^{64}$

Subfamily Pleurostomellinae Reuss, ${ }^{64}$

Genus Pleurostomella Reuss, ${ }^{64}$

Type species Dentalina subnodosa Reuss, ${ }^{129}$

\section{Pleurostomella haquei Anan ${ }^{27}$}

2019b Pleurostomella haquei Anan, ${ }^{27}$ p. 175, pl. 1, fig. 10

Remarks: This Eocene Pakistanian species has elongate lax biserial test, and the final uniserial chamber inflated and nearly globular.

\section{Pleurostomella osmani Anan ${ }^{27}$}

2019b Pleurostomella osmani Anan, ${ }^{27}$ p. 176, pl. 2, fig. 15.

Remarks: Maastrichtian Egyptian species has short test with subterminal elliptical aperture without tooth.

Superfamily Discorbacea Ehrenberg, ${ }^{43}$

Family Bagginidae Cushman, ${ }^{6}$

Subfamily Baggininae Cushman, ${ }^{62}$

Genus Sakhiella Haque, ${ }^{38}$

Type species Sakhiella nammalensis Haque, ${ }^{38}$

\section{Sakhiella nammalensis Haque ${ }^{38}$}

1956 Sakhiella nammalensis Haque, ${ }^{38}$ p. 155, pl. 10, fig. 1.

2002 Sakhiella nammalensis; Al-Hitmi, ${ }^{130}$ p. 49, pl. 3, fig. 10.

2019a Sakhiella nammalensis; Anan, ${ }^{26}$ p. 32, pl. 1, fig. 10

Remarks: This Paleocene-early Eocene species has biconvex trochospiral test but with spiral side completely involute with an extending aperture from umbilicus to the periphery covered with a distinct umbilical flap. It was recorded from Pakistan, and Qatar.

Superfamily Discorbacea Ehrenberg, ${ }^{43}$

Family Bagginidae Cushman, ${ }^{62}$

Subfamily Baggininae Cushman, ${ }^{62}$

Genus Baggina Cushman, ${ }^{100}$

Type species Baggina californica Cushman, ${ }^{100}$

Baggina eocaenica Hewaidy \& Al-Hitmi ${ }^{31}$ (Pl. 2, fig. 69)

1994 Baggina eocaenica Hewaidy \& Al-Hitmi, ${ }^{31}$ p. 19, fig. 6. 9-11.

Remarks: This early Eocene species was recorded from Dukhan Field, Qatar.

Genus Valvulineria Cushman, ${ }^{100}$

Type species Valvulineria californica Cushman, ${ }^{100}$

Valvulineria aegyptiaca LeRoy ${ }^{4}$ (P1. 2, fig. 70)

1953 Valvulineria aegyptiaca LeRoy, ${ }^{4}$ p. 53, pl. 9, figs. 21-23.

1993a Valvulineria aegyptiaca; Anan, ${ }^{112}$ p. 316.

2008 Valvulineria aegyptiaca; Anan, ${ }^{13}$ p. 364, pl. 1, fig. 5.

2012 Valvulineria aegyptiaca; Ismail, ${ }^{57}$ p. 39, pl. 3, figs. 18,19.

Remarks: This Paleocene-early Eocene species was recorded from north and central Egypt, and also from UAE.

Valvulineria critchetti LeRoy ${ }^{4}$ (Pl. 2, fig. 71)

1953 Valvulineria critchetti LeRoy, ${ }^{4}$ p. 53, pl. 3, figs. 20-22.

1992 Valvulineria critchetti; Ismail, ${ }^{128}$ p. 236, pl. 2, fig. 2.

2011 Valvulineria critchetti; Aly et al., ${ }^{28}$ p. 108, pl. 6, fig. 2.

Remarks: This early Eocene species was recorded from the north and central Egypt. It seems confined in Egypt.

\section{Valvulineria nammalensis Haque $\mathrm{e}^{38}$}

1956 Valvulineria nammalensis Haque, ${ }^{38}$ p. 159, pl. 7, fig. 10.

1993a Valvulineria sp. Anan, ${ }^{112}$ p. 316, pl. 3, fig. 3.

2019a Valvulineria nammalensis; Anan, ${ }^{26}$ p. 32, pl. 1, fig. 11a-d.

Remarks: This Paleocene-early Eocene species has two and a half dorsal whorl, 9-10 ventral chambers, and interiomarginal umbilicalextraumbilical aperture with broad thin apertural flap projecting over the umbilicus. It was recorded from Pakistan and UAE.

Valvulineria pseudotumeyensis Futyan, ${ }^{29}$ (Pl. 3, fig. 72)

1976 Valvulineria pseudotumeyensis Futyan, ${ }^{29}$ p. 531, pl. 83, figs. $2-4$.

Remarks: This early Eocene species is similar in shape to Eponides elevatus (Plummer), but much smaller (half diameter), subconical test and an angular periphery. It was recorded from Muwaqqar and Wadi Arab sections, Jordan.

Family Discorbidae Ehrenberg, ${ }^{43}$

Genus Discorbis Lamarck, ${ }^{66}$ 
Type species Discorbis vesicularis Lamarck, ${ }^{66}$

\section{Discorbis globiformis Haque ${ }^{40}$}

1962 Discorbis globiformis Haque, ${ }^{40}$ p. 21, pl. 1, figs. 3-5.

2002 Discorbis globiformis; Al-Hitmi, ${ }^{130}$ p. 49, pl. 3, fig. 11.

2019a Discorbis globiformis; Anan, ${ }^{26}$ p. 32, pl. 1, fig. 12a-c.

Remarks: This Maastrichtian-early Eocene species has gradually inflated chambers, highly trochospiral coil, umbilical region wide and covered by a long flap with smooth surface. It is recorded, so far, from Pakistan and Qatar.

Genus Trochulina d'Orbigny, ${ }^{45}$

Type species Rotalina turbo d'Orbigny, ${ }^{92}$

Trochulina aegyptiaca (LeRoy ${ }^{4}$ ( Pl. 3, fig. 73)

1953 Rotorbinella aegyptiaca LeRoy, ${ }^{4}$ p. 48, pl. 7, figs 1-3.

2008 Trochulina aegyptiaca; Anan, ${ }^{13}$ p. 365.

Remarks: Loeblich \& Tappan ${ }^{42}$ considered the genus Rotorbinella Bandy as a junior synonym of the genus Trochulina d'Orbigny. This Eocene species was recorded only in Farafra area of Egypt.

Trochulina lenticularis Hewaidy \& Al-Hitmi ${ }^{31}$ (Pl. 3, fig. 74)

1994 Trochulina lenticularis Hewaidy \& Al-Hitmi, ${ }^{31}$ p. 20, fig. 7. $1-3$.

Remarks: This early Eocene species was recorded from Dukhan Field, Qatar

Family Eponididae Hofker, ${ }^{131}$

Genus Eponides de Montfort, ${ }^{132}$

Type species Nautilus repandus Fichtel \& Moll, ${ }^{133}$

Eponides mariei Said \& Kenawy, ${ }^{5}$ (P1. 3, fig. 75)

1956 Eponides mariei Said \& Kenawy, ${ }^{5}$ p. 148, pl. 5, fig. 2.

1995 Oridorsalis mariei; Nomura \& Brohi, ${ }^{41}$ p. 229, pl. 2, fig. 2.

2012a Eponides mariei; Anan, ${ }^{18}$ p. 25, pl. 1, fig. 13.

Remarks: It was recorded from the Maastrichtian-Danian rocks of Giddi section of Sinai, and later in Paleocene of Pakistan.

Family Discorbidae Ehrenberg, ${ }^{43}$

Genus Neoeponides Reiss, ${ }^{131}$

Type species Rotalia schreibersii d'Orbigny, ${ }^{134}$

Neoeponides duwi (Nakkady¹) (Pl. 3, fig. 76)

1950 Discorbis pseudoscopos var. duwi Nakkady, ${ }^{1}$ p. 688, pl. 90, figs. 5-7.

1998 Discorbis pseudoscopos duwi; Anan, ${ }^{8}$ p.373, fig.3. 11.

2003 Neoeponides duwi; Speijer, ${ }^{135}$ p. 147, pl. 1, figs. 2-7.

2009b Neoeponides duwi; Anan, ${ }^{14}$ p. 40, pl. 1, fig. 11.

Remarks: Nakkady ${ }^{1}$ originally recorded this taxon from the base of the post-Cretaceous shales of Duwi section, Egypt, and later from northeast Sinai, Kharga Oasis and Red Sea coastal area of Egypt. Anan ${ }^{8}$ proposed the Discorbis p. pseudoscopos - D. p. duwi lineage from the Cretaceous ancestor pseudoscopos to the Paleocene descendent $d u w i$. On the other hand, Speijer ${ }^{135}$ considered duwi appears to have no phylogenetic relationship with pseudoscopos, but belongs to the genus Neoeponides due to his revision of the characters of Nakkady's holotype, and also raised the taxonomic rank of subspecies $d u w i$ to the species rank: $N$. duwi.

Superfamily Discorbinellacea Sigal, ${ }^{136}$

Family Parrelloididae Hofker, ${ }^{131}$

Genus Cibicidoides Thalmann, ${ }^{137}$

Type species Truncatolina mundula Brady, Parker \& Jones, ${ }^{138}$

Cibicidoides loeblichi (Said \& Kenawy ${ }^{5}$ ) (Pl. 3, fig. 77)

1956 Cibicides loeblichi Said \& Kenawy, ${ }^{5}$ p. 155, pl. 7, fig. 11.

2012a Cibicidoides loeblichi; Anan, ${ }^{18}$ p. 25, pl. 1, fig. 15.

Remarks: Among other characters, the biconvexity of the genus Cibicidoides make it differs from the planoconvex test of Cibicides de Montfort. This species was originally recorded from the Paleocene of Nekhl section (north Sinai), and later from Taba section (central east Sinai), Egypt. It seems, so far, it is confined in Egypt.

Superfamily Discorbinellacea Sigal, ${ }^{136}$

Family Parrelloididae Hofker, ${ }^{131}$

Genus Cibicidoides Thalmann, ${ }^{137}$

Type species Truncatulina mundula Brady, Parker \& Jones, ${ }^{138}$

Cibicidoides beadnelli (LeRoy ${ }^{4}$ ) (Pl. 3, fig. 78)

1953 Cibicides beadnelli LeRoy, ${ }^{4}$ p. 23, pl. 10, figs 6-8.

1988 Cibicides beadnelli; Keller, ${ }^{101}$ p. 165, fig. 4.

2008 Cibicidoides beadnelli; Anan, ${ }^{13}$ p. 365.

Remarks: Among other characters, the biconvexity of the genus Cibicidoides Thalmann make it differs from the planoconvex test of the Cibicides de Montfort. This Eocene species C. beadnelli was recorded in the north and central Egypt, Tunisia, Libya, Mali and Spain.

\section{Cibicidoides decoratus (LeRoy ${ }^{4}$ (Pl. 3, fig. 79)}

1953 Cibicides decoratus LeRoy, ${ }^{4}$ p. 23, pl. 6, figs 15-17.

1994 Cibicides decoratus; Speijer, ${ }^{117}$ p. 114, pl. 2, fig. 3.

2003 Cibicides decoratus; Ali, ${ }^{45}$ pl. 10, figs. 15-17.

2008 Cibicidoides decoratus; Anan, ${ }^{13}$ p. 365, pl. 1, fig. 6 .

Remarks: This Paleocene-early Eocene species was recorded from north and central Egypt.

Cibicidoides farafraensis (LeRoy ${ }^{4}$ (Pl. 3, fig. 80)

1953 Cibicides farafraensis LeRoy, ${ }^{4}$ p. 24, pl. 10, figs 1-3.

1956 Cibicidoides farafraensis; Said \& Kenawy, ${ }^{5}$ p.154, pl. 7, fig. 6.

1985 Cibicidoides farafraensis; Luger, ${ }^{58}$ p. 112, pl. 9, fig. 3. 2008 Cibicidoides farafraensis; Anan, ${ }^{13}$ p. 365, pl. 1, fig. 7. 
2011 Cibicides farafraensis; Aly et al.., ${ }^{28}$ p. 112, pl. 7, fig. 4.

2012 Cibicidoides farafraensis; Youssef \& Taha, ${ }^{59}$ pl. 5, figs. 11, 12.

Remarks: Berggren \& Aubert ${ }^{80}$ suggested that $C$. howelli (Toulmin) has closer relationship with $C$. farafraensis and seems to be equivalent. The figured $C$. howelli of Berggren $\&$ Aubert ${ }^{80}$ with very acute periphery of the whole test including the last chamber differs from the semi-rounded last chamber periphery of $C$. farafraensis. These two species are treated here to be different. The early Eocene C. farafraensis was recorded in north and central Egypt. It seems, so far, be confined in Egypt.

Cibicidoides libycus (LeRoy ${ }^{4}$ ) (P1. 3, fig. 81)

1953 Cibicides libycus LeRoy, ${ }^{4}$ p. 24, pl. 5, figs. 1-3.

1980 Heterolepa libyca; Saperson \& Janal, ${ }^{139}$ p. 404, pl. 2, fig. 4.

2001 Heterolepa libyca; Hewaidy \& Strougo, p. 17, pl. 2, figs 25, 26.

2005 Heterolepa libyca; Sztrákos, ${ }^{140}$ p. 189, pl. 9, fig. 9 .

2008 Cibicidoides libycus; Anan, ${ }^{13}$ p. 365, pl. 1, fig. 8.

2012 Heterolepa libyca; Ismail,,$^{57}$ p. 41, pl. 3, fig. 29

Remarks: According to Loeblich \& Tappan ${ }^{42}$ the genus Heterolepa has a slit-like aperture extending about half of the distance to umbilicus and extending short distance across periphery on dorsal side, but without biumbonate test. $H$. libyca differs from the genus Cibicidoides Thalmann by its biconvexity among other characters. Both Cibicidoides alleni and C. libycus have conspicuous biumbonate test, as well as a low interiomarginal equatorial arch aperture. The Paleocene-early Eocene C. libycus is characterized by its depressed sutures in both sides than elevated and taper sutures in the dorsal side and slightly in the ventral side in C. alleni (Plummer). C. libycus was recorded from Egypt and later from Libya, Tunisia, France, Turkmenia, North and South Atlantic.

\section{Cibicidoides nammalensis Haque $^{58}$}

1956 Cibicidoides nammalensis Haque ${ }^{58}$ p. 205, pl. 20, fig. 10.

2005 Cibicidoides nammalensis; Sztrákos, ${ }^{140}$ p. 226, pl. 15, fig. 35.

2019a Cibicidoides nammalensis; Anan, ${ }^{26}$ p. 32, pl. 2, fig. 14a-c.

Remarks: This early Eocene species has biconvex test with keel, about 10 chambers in the ventral side with umbo. It was recorded from Pakistan, France and Iraq.

Cibicidoides pharaonis (LeRoy ${ }^{4}$ (Pl. 3, fig. 82)

1953 Cibicides pharaonis LeRoy, ${ }^{4}$ p. 24, pl. 7, figs 9-11.

1988 Cibicidoides pharaonis; Anan \& Sharabi ${ }^{81}$ p. 215. pl. 2, fig. 20

2001 Cibicidoides pharaonis; Hewaidy \& Strougo, ${ }^{52}$ p. 17, pl. 2, figs 27,28 .

2007 Cibicidoides pharaonis; Alegret \& Ortiz, ${ }^{115}$ p. 440, pl. 1, fig. 24.

2008 Cibicidoides pharaonis; Anan, ${ }^{13}$ p. 366, pl. 1, fig. 9.

Remarks: This Paleocene-early Eocene species was recorded from north and central Egypt.
Cibicidoides pseudoacutus (Nakkady') (Fig. 5, fig. 83)

1950 Anomalina pseudoacuta Nakkady, ${ }^{1}$ p. 691, pl. 90, figs. 29-32. 1994 Cibicidoides pseudoacutus; Speijer ${ }^{117}$ p. 54, pl. 7, fig. 6, p. 158, pl. 4, fig. 2.

2002 Cibicidoides pseudoacuta; Galeotti \& Coccioni, ${ }^{141}$ p. 198, fig. 1.

2005 Cibicidoides pseudoacuta; Guasti et al., ${ }^{142}$ p. 4, pl. 1, fig. 14. 2005 Gavelinella pseudoacuta; Sztrákos, ${ }^{140}$ p. 214, pl. 9, fig.14. 2006 Gavelinella pseudoacuta; Ernst et al., ${ }^{143}$ p. 98, pl. 1, figs. j, k. 2007 Cibicidoides pseudoacuta; Alegret \& Ortiz, ${ }^{115}$ p. 440, pl. 2, fig. 2.

2012 Anomalina pseudoacuta; Ismail, ${ }^{57}$ p. 40, pl. 3, fig. 24.

Remarks: The Paleocene-Eocene species is characterized by its fine beads, which surround a thick irregular spiral of translucent shell substance on ventral side. It was originally described be Nakkady ${ }^{1}$ from the Cretaceous Chalk and post-Cretaceous in central Egypt. Later on, it is was recorded from Paleocene-Eocene in Egypt.

Superfamily Planorbulinacea Schwager, ${ }^{144}$

Family Planulinidae Bermúdez, ${ }^{145}$

Genus Planulina d'Orbigny, ${ }^{92}$

Type species Planulina ariminensis d'Orbigny, ${ }^{92}$

Planulina berggreni Anan9 (Pl. 3, fig. 84)

2001 Planulina berggreni Anan, ${ }^{9}$ p. 136, pl. 1, fig. 2.

2011 Planulina berggreni; Anan, ${ }^{17}$ p. 62, pl. 3, fig. 31.

Remarks: This Danian species has large and semicircular test with flat sides, suture extending backward and beaded around umbilical area, but depressed in the last chamber, surface smooth, aperture slitlike at peripheral end. It was recorded from Duwi, Egypt.

Superfamily Asterigerinacea d'Orbigny, ${ }^{45}$

Family Epistomariidae Hofker, ${ }^{146}$

Subfamily Epistomariinae Hofker, ${ }^{146}$

Genus Epistomaria Galloway, ${ }^{147}$

Type species Discorbina rimosa Parker \& Jones, ${ }^{148}$

Epistomaria saudica Hasson ${ }^{149}$ (P1. 3, fig. 85)

1985 Epistomaria saudica Hasson, ${ }^{149}$ p. 356, pl. 2, figs. 4-6.

Remarks: This early Eocene species was recorded from of Rub'al Khali Basin wells, Saudi Arabia (SA).

Family Alfredinidae Singh \& Kalia, ${ }^{150}$

Genus Epistomaroides Uchio, ${ }^{151}$

Type species Discorbina polystomelloides Parker \& Jones, ${ }^{148}$

Epistomaroides qatarica Hewaidy \& Al-Hitmi ${ }^{31}$ (Pl. 3, fig. 86)

1994 Epistomaroides qatarica Hewaidy \& Al-Hitmi, ${ }^{31}$ p. 22, fig. 7. 7,8 .

Remarks: This early Eocene species was recorded from Ummer 
Radhuma Formation of Qatar.

Superfamily Nonionacea Schultze, ${ }^{152}$

Family Nonionidae Schultze, ${ }^{152}$

Subfamily Nonioninae Schultze, ${ }^{152}$

Genus Nonion de Montfort, ${ }^{132}$

Type species Nonion faba Fichtel \& Moll, ${ }^{133}$

Nonion fayoumensis Ansary ${ }^{51}$ (P1. 3, fig. 87)

1954 Nonion fayoumensis Ansary, ${ }^{51}$ p. 317, pl. 1, fig. 4.

2007 Nonion fayoumensis; Farouk, ${ }^{153}$ p. 64, pl. 2, fig. 15.

2013a Nonion fayoumensis; Anan, ${ }^{20}$ p. 54, pl. 1, figs. 18-20.

Remarks: This species was originally described from Eocene of Fayoum, and later from Sinai, Egypt.

\section{Nonion maadiensis Ansary ${ }^{51}$ (Pl. 3, fig. 88)}

1954 Nonion maadiensis Ansary, ${ }^{51}$ p. 318, pl. 1, fig. 5.

2013a Nonion maadiensis; Anan, ${ }^{20}$ p. 54, pl. 1, figs. 21-24.

Remarks: This Eocene species was originally described from Maadi area, and later from Qattamiya (Greater Cairo) and Sinai, Egypt. The Nonion sp. of Farouk $1^{53}$ (p. 64, pl. 2, fig. 14) is considered here belongs to $N$. maadiensis.

\section{Nonion trompi Ansary ${ }^{51}$ (Pl. 3, fig. 89)}

1954 Nonion trompi Ansary, ${ }^{51}$ p. 319, pl. 1, fig. 6.

2013a Nonion trompi; Anan, ${ }^{20}$ p. 54, pl. 1, figs. 25, 26.

Remarks: This species was originally described from the Eocene rocks of Fayoum and Maadi area, Egypt.

Genus Nonionella Cushman, ${ }^{100}$

Type species Nonionella miocenica Cushman, ${ }^{100}$

Nonionella africana LeRoy ${ }^{4}$ (Pl. 3, fig. 90)

1953 Nonionella africana LeRoy, ${ }^{4}$ p. 42, pl.10, figs. 9-11.

1956 Nonionella africana; Haque, ${ }^{38}$ p. 114, pl. 6, figs. 1, 9.

2008 Nonionella africana; Anan, ${ }^{13}$ p. 366, pl. 1, fig. 10.

2011 Nonionella africana; Aly et al. ${ }^{28}$ p. 113, pl. 7, fig. 8 .

Remarks: This Paleocene-Eocene species was recorded only in central Egypt and Pakistan.

\section{Nonionella haquei Anan ${ }^{26}$}

1956 Nonionella sp. Haque, ${ }^{38}$ p. 156, pl. 7, fig. 21.

1956 Nonionella sp. Said \& Kenawy, ${ }^{5}$ p. 156, pl. 7, fig. 21.

1960 Nonionella sp. Haque, ${ }^{39}$ p. 24, pl. 6, fig. 2

2019a Nonionella haquei Anan, ${ }^{26}$ p. 33, pl. 2, fig. 15a-f.

Remarks: This Paleocene-Eocene species has large biconvex test but not symmetrically developed, 12 chambers in the last formed whorl, a slit aperture on the periphery at the base of the last chamber. It was recorded, so far, from Pakistan and Egypt.
Superfamily Chilostomellacea Brady, ${ }^{154}$

Family Alabaminidae Hofker, ${ }^{131}$

Genus Alabamina Toulmin, ${ }^{155}$

Type species Alabamina wilcoxensis Toulmin, ${ }^{155}$

Alabamina cavelieri Hewaidy \& Al-Hitmi, ${ }^{31}$ (Pl. 3, fig. 91)

1994 Alabamina cavelieri Hewaidy \& Al-Hitmi, ${ }^{31}$ p. 23, fig. 7. 9-14.

Remarks: This early Eocene species was recorded from Umm er Radhuma Formation of Qatar.

Superfamily Chilostomellacea Brady, ${ }^{154}$

Family Osangulariidae Loeblich \& Tappan, ${ }^{156}$

Genus Osangularia Brotzen, ${ }^{157}$

Type species Osangularia lens Brotzen, ${ }^{157}$

Osangularia desertorum (LeRoy) (P1. 3, fig. 92)

1953 Parrella desertorum LeRoy, ${ }^{4}$ p. 43, pl. 3, figs. 17-19.

2008 Osangularia desertorum; Anan, ${ }^{13}$ p. 366.

Remarks: Loeblich \& Tappan ${ }^{42}$ considered the genus Parrella Finlay fall within the species concept of the genus Osangularia Brotzen. This early Eocene species was recorded only in central Egypt.

Superfamily Chilostomellacea Brady, ${ }^{154}$

Family Heterolepidae Gonzáles-Donoso, ${ }^{158}$

Genus Anomalinoides Brotzen, ${ }^{159}$

Type species Anomalinoides plummerae Brotzen, ${ }^{159}$

Anomalinoides aegyptiacus (LeRoy ${ }^{4}$ (Pl. 3, fig. 93)

1953 Anomalina aegyptiaca LeRoy, ${ }^{4}$ p. 17, pl. 7, figs 21-23.

1994 Anomalinoides aegyptiacus; Speijer, ${ }^{117}$ p. 116, pl. 5, fig. 3.

2001 Anomalinoides aegyptiacus; Hewaidy \& Strougo, ${ }^{52}$ p. 17, pl. 2, figs 33,34 .

2007 Anomalinoides aegyptiacus; Alegret \& Ortiz, ${ }^{115}$ p. 438, pl. 1, fig. 13 .

2011 Anomalinoides aegyptiacus; Aly et al., ${ }^{28}$ p. 116, pl. 8, fig. 1.

Remarks: Loeblich \&Tappan ${ }^{42}$ noted that the type specimen of Anomalina was lost; a petition was submitted to the ICZN for the suppression of it and retention of Epistomaroides Uchio $^{151}$ as a valid genus. Anan ${ }^{7}$ noted that the characters of fayoumensis species belongs to the genus Anomalinoides. This Paleocene-early Eocene species was recorded only in central Egypt.

Anomalinoides esnehensis (Nakkady') (Pl. 3, fig. 94)

1950 Anomalina scrobiculata (Schwager) var. esnehensis Nakkady, ${ }^{1}$ p.691, pl. 90, figs. 33, 34 .

2009b Anomalinoides esnehensis; Anan, ${ }^{14}$ p. 42.

Remarks: Nakkady ${ }^{1}$ recorded this species from the Paleocene shales of Sinai, Luxor and Duwi sections, Egypt. 


\section{Anomalinoides desertorum (LeRoy ${ }^{4}$ (Pl. 3, fig. 95)}

1953 Anomalina desertorum LeRoy, ${ }^{4}$ p.17, pl.7, figs.18-20.

1993 Anomalina desertorum Boltovskoy \& Vera Ocampo, ${ }^{116} \mathrm{p}$. 146.

2008 Anomalinoides desertorum; Anan, ${ }^{13}$ p. 367, pl. 1, fig. 11.

Remarks: The Late Paleocene-early Eocene A. desertorum was recorded in central Egypt, and also Arabian Sea.

Anomalinoides fayoumensis (Ansary, ${ }^{51}$ ) (P1. 3, fig. 96)

1954 Anomalina fayoumensis Ansary, ${ }^{51}$ p. 325, pl. 1, fig. 11.

2010a Anomalinoides fayoumensis; Anan, ${ }^{35}$ p. 170, pl. 2, fig. 5.

2011 Anomalinoides fayoumensis; Aly et al., ${ }^{28}$ p. 116, pl. 8, fig. 5.

2013a Anomalinoides fayoumensis; Anan, ${ }^{20}$ p. 55, pl. 1, figs. 2729.

Remarks: This species was recorded from the Eocene rocks of Fayoum area, and later from Mokattam area ( Greater Cairo), Nile Valley and Siwa area of Egypt. It was also recorded in Jordan and UAE.

\section{Anomalinoides leroyi Anan, ${ }^{13}$ (Pl. 3, fig. 97)}

1953 Anomalina sp. K LeRoy, ${ }^{4}$ p. 19, pl. 3, figs 26-28.

2008 Anomalinoides leroyi Anan, ${ }^{13}$ p. 367, pl. 1, fig. 12.

2011 Anomalinoides leroyi; Anan, ${ }^{17}$ p. 63, pl. 3, fig. 32.

Remarks: This latest Maastrichtian to Danian species was described from the Duwi section, central Egypt.

Anomalinoides zitteli (LeRoy ${ }^{4}$ (Pl. 3, fig. 98)

1953 Cibicides zitteli LeRoy, ${ }^{4}$ p. 25, pl. 6, figs 20-22.

1994 Anomalinoides zitteli; Speijer, ${ }^{117}$ p. 118, pl. 5, fig. 1.

2001 Anomalinoides zitteli; Hewaidy \& Strougo, ${ }^{52}$ p. 17, pl. 2, figs $29,30$.

2007 Anomalinoides zitteli; Alegret \&Ortiz, ${ }^{115}$ p. 439, pl. 1, fig. 18.

Remarks: According to Loeblich \& Tappan ${ }^{42}$ the genus Anomalinoides Brotzen is distinguished by its low trochospiral test, central boss in spiral side and broad rounded periphery, while the genus Cibicidoides Thalmann has biconvex, biumbonate and trochospiral test, limbate curved suture on the spiral side and angular periphery. For that, the species zitteli belongs here to the genus Anomalinoides. This Paleocene-early Eocene species was recorded from north and central Egypt.

Family Gavelinellidae Hofker, ${ }^{160}$

Subfamily Gyroidinoidinae Saidova, ${ }^{109}$

Genus Gyroidinoides Brotzen, ${ }^{161}$

Type species Rotalina nitida Brotzen, ${ }^{161}$

Gyroidinoides aegyptiaca (Ansary ${ }^{51}$ ) (Pl. 3, fig. 99)

1954 Gyroidina aegyptiaca Ansary, ${ }^{51}$ p. 324, pl.1, fig.1.

2013a Gyroidinoides aegyptiaca; Anan, ${ }^{20}$ p. 55, pl. 1, figs. 30-33.

Remarks: It was described from the Eocene of Fayoum, later from Qattamiya area, Egypt.
Gyroidinoides luterbacheri Anan, ${ }^{162}$ (Pl. 3, fig. 100)

2004 Gyroidinoides luterbacheri Anan, ${ }^{162}$ p. 49, pl. 1, fig. 13.

2011 Gyroidinoides luterbacheri; Anan, ${ }^{17}$ p. 63, pl. 3, fig. 33.

Remarks: This Paleocene species was described from the DanianSelandian of Duwi section, Egypt.

Genus Angulogavelinella Hofker, ${ }^{163}$

Type species Discorbina gracilis Marsson, ${ }^{69}$

Angulogavelinella bandata Futyan, ${ }^{29}$ (Pl. 3, fig. 101)

1976 Angulogavelinella bandata Futyan, ${ }^{29}$ p. 534, pl. 83, figs. 1-3.

Remarks: This Paleocene species was founded in the Danian of Tell Burma, south Jordan.

Family Rotaliidae Ehrenberg, ${ }^{164}$

Subfamily Culvillierininae Loeblich \& Tappan, ${ }^{156}$

Genus Ornatanomalina Haque, ${ }^{38}$

Type species Ornatanomalina geei Haque, ${ }^{38}$

Ornatanomalina ennakhali Anan, ${ }^{17}$ (Pl. 3, fig. 102)

1996 Ornatanomalina sp. Anan, ${ }^{165}$ p. 154, fig. 4. 10.

2011Ornatanomalina ennakhali; Anan, ${ }^{17}$ p. 63, pl. 3, fig. 34.

2013b Ornatanomalina ennakhali; Anan, ${ }^{166}$ p. 115, pl. 3, fig. 5.

Remarks: This late early Eocene species differs from the type species $O$. geei and its subspecies $O$. geei compressa and other six Haque's species and subspecies ${ }^{38,39}$ from Pakistan $(O$. crookshanki, $O$. c. rugosa. O. glaessneri, O. hafeezi, O. elegantula and O. pustulosa) by its discontinuous ribs, not rounded periphery and lacking the radial median ridges across the chamber surface. O. ennakhali Anan was recorded in the Ypresian of the Rus Formation, J. Hafit in UAE, and this contribution expands the lithostratigraphic level of the early Eocene rocks in Arabia from the upper part of Umm Er Radhuma Formation (in SA and Qatar) to Rus Formation (in UAE), but the latter formation falls within the lower Lutetian rather than the upper Ypresian by Hasson. ${ }^{30}$

\section{Ornatanomalina hafeezi Haque, ${ }^{38}$}

1956 Ornatanomalina hafeezi Haque, ${ }^{38}$ p. 201, pl. 18, fig. 6.

2019a Ornatanomalina hafeezi; Anan, ${ }^{26}$ p. 33, pl. 2, fig. 17.

Remarks: This Pakistanian genus was considered by Loeblich \& Tappan ${ }^{42}$ as a senior synonym of the Saudian genus Saudella of Hasson (1985) and its representatives. This Eocene species was recorded from Pakistan, Saudi Arabia, Qatar (by Hewaidy ${ }^{167}$ ) and Nigeria (by Haynes \& Nwabufo-Ene ${ }^{168}$ ).

\section{Ornatanomalina inflata $\left(\right.$ Hasson $\left.^{30}\right)(\mathrm{Pl} .3$, fig. 103)}

1985 Saudella inflata Hasson, ${ }^{30}$ p. 350, pl. 2, figs. 1-3.

Remarks: Loeblich \& Tappan ${ }^{42}$ considered the genus Ornatanomalina Haque from Pakistan as a senior synonym of Saudella Hasson $^{30}$ from SA, and considered $O$. hafeezi as a junior synonym of $O$. geei (the type species of the genus Ornatanomalina). This planispiral species was recorded from early Eocene Umm er Radhuma Formation in wells of Rub'al Khali Basin, Saudi Arabia (SA). 


\section{Ornatanomalina ornata (Hasson ${ }^{30}$ ) (P1. 3, fig. 104)}

1985 Saudella ornata Hasson, ${ }^{30}$ p. 348, pl. 1, figs. 6-9.

Remarks: This species has eleven chambers visible in the last whorl, periphery rounded in later chambers and blunt in earlier part of test, apertural face smooth bordered by a raised imperforate rim. It was recorded also from early Eocene of Umm er Radhuma Formation in the Rub'al Khali Basin wells, Saudi Arabia (SA).

Ornatanomalina porosa $\left(\operatorname{Hasson}^{30}\right)$ (Pl. 3, fig. 105)

1985 Saudella porosa Hasson, ${ }^{30}$ p. 350, pl. 1, figs. 10-12.

Remarks: This species was recorded from early Eocene of SA.

Ornatanomalina rugosa $\left(\right.$ Hasson $\left.^{30}\right)$ (Pl. 3, fig. 106)

1985 Saudella rugosa Hasson, ${ }^{30}$ p. 348, pl. 1, figs. 1-5.

Remarks: This species was recorded from early Eocene of SA.

Subfamily Pararotaliinae Reiss, ${ }^{169}$

Genus Pararotalia Le Calves, ${ }^{170}$

Type species Rotalia inermis Terquem, ${ }^{171}$

\section{Pararotalia khirthari Haque, ${ }^{39}$}

1960 Pararotalia khirthari Haque, ${ }^{39}$ p. 3, pl. 6, fig. 5.

2010a Pararotalia khirthari; Anan, ${ }^{35}$ p. 171.

2019a Pararotalia khirthari; Anan, ${ }^{26}$ p. 33, pl. 2, fig. 18.

Remarks: This middle-late Eocene species has low trochospiral test with axially pointed long spine for each chamber. It was recorded, so far, from Pakistan and UAE.

Family Elphidiidae Galloway, ${ }^{120}$

Subfamily Elphidiinae Galloway, ${ }^{120}$

Genus Elphidiella Cushman, ${ }^{172}$

Type species Polystomella arctica Parker \& Jones in Brady, ${ }^{173}$

Elphidiella africana (LeRoy ${ }^{4}$ (P1. 3, fig. 107)

1953 Elphidium africanum LeRoy, ${ }^{4}$ p. 28, pl. 3, figs 11, 12.

1998 Elphidium africanum; Haynes \& Nwabufo-Ene, ${ }^{168}$ p. 53.

2008 Elphidium africanum; Anan, p. 367.

Remarks: This Eocene species belongs here to the genus Elphidiella as treated previously by Berggren ${ }^{174}$ and Haynes \& Nwabufo-Ene. ${ }^{168}$ It was recorded from Egypt, as well as Libya, Mali and Nigeria (central Africa).

\section{Genus Elphidium de Montfort, ${ }^{132}$}

Type species Nautilus macellus Fichtel \& Moll, ${ }^{133}$

Elphidium cherifi Anan, ${ }^{35}$ (P1. 3, fig. 108)

2010a Elphidium cherifi Anan, ${ }^{35}$ p. 172, pl. 2, fig. 8.

2011 Elphidium cherifi; Anan, ${ }^{17}$ p. 64, pl. 3, fig. 35.

Remarks: This middle to late Eocene species differs from the figured form E. leave of Cherif et al., ${ }^{118}$ (p. 52, pl. 4, fig. 18) by its backward extensions of the chambers in J. Hafit. It was recorded from late Eocene of J. Hafit, UAE.

\section{Paleogeography}

The paleogeographic maps (partly or regionally) of Berggren, ${ }^{175}$ Mintz, ${ }^{176}$ Rosenbaum et al., ${ }^{177}$ show the Tethyan realm had been connected with the Indo-Pacific Ocean from the east and Atlantic Ocean to the west via Mediterranean Sea crossing the Middle East region during the Paleogene time (Figure 3). It is proved by the existence of the identified taxa from the Middle East in other localities in the Tethys. Accordingly, the following remarks can be added:

i. Berggren ${ }^{175}$ suggested that during the Paleogene, the fauna of the Mediterranean and the Indo-Pacific exhibit pronounced similarities, which indicate that the connection between the two areas was mentioned by a marine seaway, and the east Atlantic fauna was much more closely related to the fauna than it is today. In the western Atlantic a narrow connection between it and Pacific existed.

ii. Haq \& Aubry ${ }^{178}$ noted that the North Africa and Middle East formed important parts of the Tethyan link between the Atlantic and Pacific Oceans during Paleogene.

iii. Anan \& Sharabi ${ }^{81}$ noted that the genus Orthokarstenia is recorded only in the central and southern Egypt. Anan ${ }^{8,22}$ concluded that all recorded members of the Maastrichtian-early Eocene genus Orthokarstenia in Egypt (O. applinae, O. esnehensis, $O$. eleganta, O. higazyi, O. nakkadyi) have been restricted from the north to the south of Lat. $27^{\circ} \mathrm{N}$ in Egypt. Anan ${ }^{22}$ recorded his Paleocene-early Eocene species Orthokarstenia nakkadyi, so far, from many sites in the southern Tethys (Egypt and Tunisia) and also the northern Tethys (Spain and France).

iv. Anan ${ }^{179}$ concluded that the Tethyan realm during the middle-late Eocene extends to the southeast and connected with the IndoPacific realm via seaway separating Arabia from Iran-India region.Haynes \& Nwabufo-Ene ${ }^{168}$ suggested wider Tethyan connections, as far as the Carpathian and Pakistan.

v. Rögl ${ }^{180}$ noted that by the end of the Eocene the Tethys Ocean had already vanished, a new Indian Ocean was born, the western end of the Tethys was reduced to a Mediterranean Sea, Europe was still an archipelago and intercontinental seas covered large areas of the European platform and of western Asia. Between the stable Eurasian platform and the relics of the western Tethys, an elongate deep basins had formed and north of India a marine connection stretched to the west Pacific. Moreover, an important connection of the Tethys with the Polar Sea existed via the Turgai Strait, on the far side of the Oral Mts. These seaways around Asia and the connections with the Polar Sea enable warm-water exchanges and probably explain the sustained warm climate during the Late Eocene. For that the western end of relic Tethys connected Indo-Pacific and Atlantic Oceans.

vi. Meulenkamp \& Sissingh ${ }^{181}$ noted that the Arabian Platform, still largely covered by the sea in early to middle Eocene times, was subject to a major regression in the middle to late Eocene.

The following paleontological remarks can be added:

a. Most of the identified calcareous foraminiferal species from the Maastrichtian-Paleogene in the Middle East were erected from Egypt (84/119, about $70.5 \%$ ), 8 from Jordan (about $6.7 \%$ ), 8 from UAE (about $6.7 \%$ ), 5 from Saudi Arabia (about 4.2\%), 4 from Qatar (about $3.3 \%$ ) and 8 from Pakistan (about $6.7 \%$ ). 
b. The number of species were erected from the Middle East are 119 species: $32 / 119$ species belong to Anan, ${ }^{7-10,13,15,17,23-27,34-37}$ 24 species to LeRoy, ${ }^{4} 12$ species to Ansary, ${ }^{50} 12$ species to Nakkady, ${ }^{1-3} 8$ species to Futyan, ${ }^{29} 8$ species to Haque, ${ }^{38-40}$ 6 species to Said \& Kenawy, ${ }^{5} 6$ species to Abdou \& Abdel Kireem, ${ }^{6} 5$ species to Hasson, ${ }^{30} 4$ species to Hewaidy \& AlHitmi, ${ }^{31} 1$ species for each:, Helal ${ }^{78}$ and Aly et al. ${ }^{28}$

c. Nine of the recorded species are observed in Tunisia, 3 in Libya, 3 in Spain, 3 in Atlantic Ocean, 3 in France and 1 in Arabian Sea.

d. Seventy five species of the identified species, so far, are endemic to Egypt.

e. Thirteen species of the Egyptian species are found also in other Tethyan localities, outside Arabia: Pakistan, Arabian Sea, Libya, Tunisia, France, Spain, Atlantic Ocean: Tristix aubertae, Palmula woodi, Aragonia semireticulata, Orthokarstenia nakkadyi, Bulimina farafraensis, Trifarina esnaensis, Eponides mariei, Cibicidoides beadnelli, C. libycus, C. pseudoacutus, Nonionella africana, Anomalinoides desertorum and Elphidiella africana.

f. Five species of the Saudian species, so far, are endemic to Saudi Arabia.

g. Eight species of the Jordanian species, so far, are endemic to Jordan.

h. Eight species of the Emeratian species, so far, are endemic to UAE: Laevidentalina hudae, L. salimi, Marginulinopsis karimae, Hemirobulina olae, Ramulina futyani, Turrilina hassani, Ornatanomalina ennakhali and Elphidium cherifi.

i. Four species of the Qatarian species, so far, are endemic to Qatar.

j. Nine species were recorded in both Egypt and UAE: Amphimorphina youssefi, Saracenaria leroyi, Astacolus bifurcatus, Hemirobulina bassiounii, Ramulina futyani, Uvigerinella nakkadyi, Trifarina esnaensis, Valvulineria aegyptiaca, A. fayoumensis.

k. Eight species were recorded in both Egypt and Tunisia: Tristix aubertae, Palmula woodi, Orthokarstenia nakkadyi, Bulimina farafraensis, Trifarina esnaensis, Cibicidoides beadnelli, C. libycus and C. pseudoacutus 12. Five species were recorded in both Egypt and Jordan: Frondicularia nakkadyi, Lenticulina trompi, Palmula woodi, Orthokarstenia higazyi, and Anomalinoides fayoumensis.

1. Four species were recorded in both Egypt and Libya: Eponides mariei, Cibicidoides beadnelli, C. libycus and Elphidiella africana.

$\mathrm{m}$. Six of Egyptian species have wide distribution in the Tethys (outside Arabia): Orthokarstenia nakkadyi, Bulimina farafraensis, Trifarina esnaensis, Cibicidoides beadnelli, C. libycus and C. pseudoacutus.

n. Palmula ansaryi Anan $^{7}$ has wide geographic distribution in Egypt. It described from the Bartonian of many sections in Fayoum area (Guta I, III, and Naalun) and Sinai (Tayiba) sections by Anan. ${ }^{7}$ Later on, it also recorded from the Greater (Bani Suef, Warshet ErRokham section) by Hussein ${ }^{72}$ and by Helal $^{78}$ from Cairo area (Helwan and Giza Pyramid area) and Nile Valley and in Fayoum area (El-Nazia section). This species seems confined to the Lutetian-Bartonian planktic foraminiferal zones of Berggren \& Pearson ${ }^{182}$ and equated the homogeneous benthic foraminifera $P$. ansaryi zone of Anan. ${ }^{7}$ It considered an excellent marker for the Bartonian/Priabonian (middle/late Eocene) boundary in Egypt.

o. The genus Ornatanomalina and its representatives have a wide geographic distribution in western Asia, earlier was recorded from Pakistan $\left(\mathrm{Haque}^{38}\right)$ and later from Rub' Al Khali Basin of Saudi Arabia $\left(\operatorname{Hasson}^{30}\right)$, and also in UAE after the record of the late Early Eocene species Ornatanomalina ennakhali Anan ${ }^{183}$ from J. Hafit, UAE.

p. The number differences of the recorded species between the different localities in the Middle East may be due to not detailed study, different environmental parameters and/or misidentification.

\section{Paleoecology and paleobathymetry}

a. LeRoy ${ }^{4}$ noted that in certain respects the microfauna of the Esna Shale of Egypt exhibits an affinity with the Paleocene Midway Type fauna (MTF) assemblages of the United States Gulf Coast area. Said \& Kenawy $^{5}$ described about 275 of foraminifera species from the Maastrichtian-Paleogene of the northern Sinai, Egypt. Berggren \& Aubert ${ }^{184}$ noted that the Paleogene of these fauna shows an affinity with the MTF (middle-outer neritic environment (50-200 m).

b. Berggren \& Aubert ${ }^{184}$ considered the faunal assemblage of Maqfi section, Farafra area and the taxa of Said \& Kenawy ${ }^{5}$ in the northern Sinai of Egypt to be predominantly related to the MTF (middle-outer neritic environment, 50-200 m).

c. Keller (1983) noted that general cooling trend between middle Eocene to early late Oligocene is indicated by the successive replacement of warm middle Eocene surface water species (planktic) by cooler Late Eocene intermediate water species. This is indicated by the coexistence of surface, intermediate and deep dwelling species group, suggest that increased thermal gradients developed between the equator and poles nearly coincident with the development of late psychrosphere.

d. Cherif \& EL Deeb ${ }^{185}$ noted that arid climate at the close of the middle Eocene became markedly wetter and seems to have been accompanied by a cooling of the water temperature. Moreover, the climatic changes inferred from the Hafit area seems to have been widespread, at least in part of the Middle East.

e. Anan \& Hewaidy ${ }^{79}$ considered the Nile Valley Facies is related to the MTF. It means that most northern and central Egypt, according to these authors, shows an affinity with the MTF, middle-outer neritic environment $(50-200 \mathrm{~m})$.

f. Anan ${ }^{179}$ noted that in the late Eocene time in the UAE and surrounding area had been located in the tropical and warmtemperate region based on many faunal environmental elements (presence of keel, accessory apertures, tubular spines in some planktic foraminiferal assemblage and high $\mathrm{P} / \mathrm{B}$ ratio).

g. Anan ${ }^{13}$ noted that barren dark shale bed that rest on the top Maastrichtian horizon (K/T) in some Tethyan localities: Ain Dabadib (Anan \& Sharabi ${ }^{81}$ ), Gabal Duwi $\left(\right.$ Anan $^{10}$ ) and also in El Kef section in Tunisia (Keller ${ }^{101}$ ) is most probably indicative of low oxygenic level in that time. 
h. Anan ${ }^{16}$ noted that his species Vaginulinopsis boukharyi is restricted in the Paleocene Tarawan Chalk of Duwi section, which may represents an endemic environment, reflects a sealevel fall, most probably, in the inner neritic environment (about $50 \mathrm{~m}$ ) than middle-outer neritic environment (about 150-200 m) of Dakhla Shale below and Esna Shale above.

i. $A n a n^{17}$ noted that the probable environment for Sinai, Egypt (represented by Abu Zenima section) is outer neritic-upper bathyal (200-400 m), which it deeper than the other sites in Egypt: the north Western Desert area (represented by Jiran El Ful section), Farafra-Bahariya area (Maqfi section) and Nile Valley in central Egypt (Duwi section), are deposited in the middle-outer neritic environment (75-200 m).

\section{Conclusions}

The analysis of the Maastrichtian-Paleogene foraminiferal species and subspecies from the Middle East led to the following conclusions:

a) The paleontology, stratigraphy and paleogeographic remarks are presented to 119 diagnostic calcareous hyaline foraminiferal species have been erected from the Maastrichtian-Paleogene rocks from Egypt, Jordan, Saudi Arabia, Qatar, UAE and Pakistan in the Middle East.

b) The number of species were erected from the Middle East are119 species: $32 / 119$ species belong to Anan, ${ }^{7-27} 24$ to LeRoy, ${ }^{4}$ 12 to Ansary, ${ }^{50} 12$ to Nakkady, ${ }^{1-3} 8$ to Futyan, ${ }^{29} 8$ to Haque, ${ }^{38-40} 6$ to Said \& Kenawy, 6 to Abdou \& Abdel Kireem, ${ }^{6} 5$ to Hasson, ${ }^{50}$ 4 to Hewaidy \& Al-Hitmi, ${ }^{31} 1$ species for each: Helal, ${ }^{78}$ and also Aly et al. ${ }^{28}$

c) Most of these identified species were erected from Egypt (84/119, about 70.5\%).16/84 species (14. $1 \%$ ) of the Egyptian species are found in other Tethyan localities, outside Arabia: Pakistan, Atlantic Ocean, Spain, France, Tunisia, Libya.

d) Two/119 species are recorded from the Maastrichtian, 9 are recorded from the Maastrichtian-Paleocene, 23 are recorded from the Paleocene, and another 23 species are recorded from the Paleocene and continue to Eocene, while 62 species belong to Eocene time.

e) Cherif \& El Deeb ${ }^{118}$ and Anan ${ }^{179}$ noted that the distribution of the identified Paleogene species from Middle East most probably represent tropical-subtropical fauna.

f) Anan \& Hamdan ${ }^{186}$ noted that an incursion of warm temperate water-mass on the foredeep was sporadic and intermittent throughout the Paleocene of J. Malaqet, UAE.

g) Anan ${ }^{17}$ noted that the probable environment for Sinai in the northern Egypt (represented by Abu Zenima section) is outer neritic-upper bathyal (200-400 m), which it deeper than the other sites in Egypt: the North Western Desert area (represented by Jiran El Ful section), Farafra Bahariya area (Maqfi) and Nile Valley (Duwi) in central Egypt, which are most probably deposited in the middle-outer neritic (75-200 m).

\section{Acknowledgments}

None.

\section{Conflicts of interest}

Author declares that there is no conflict of interest.

\section{References}

1. Nakkady SE. A new foraminiferal fauna from the Esna Shale and Upper Cretaceous chalk of Egypt. Journal of Paleontology. 1950;24(6):675-692.

2. Nakkady SE. The stratigraphic implication of the accelerated tempo of evolution in the Mesozoic-Cenozoic transition of Egypt. Journal of Paleontology. 1955;29(4):702-706.

3. Nakkady SE. Biostratigraphy of the Um Elghanayem section, Egypt Micropaleontology. 1959;5(4):453-472.

4. LeRoy LW. Biostratigraphy of Maqfi section, Egypt. Vol 54. Geological Society of American Memoir; 1953. 73 p.

5. Said R, Kenawy A. Upper Cretaceous and Lower Tertiary foraminifera from northern Sinai, Egypt. Micropaleontology. 1956;(2):105-173.

6. Abdou HF, Abdel-Kireem MR. On the occurrence of Altistoma species in the Middle Eocene of Fayoum province, Egypt. Proceedings of the Vth African Colloquium on Micropalaeontology, Addis-Ababa. Madrid: Enadimsa; 1975. 46-55 p.

7. Anan HS. Benthic foraminifera around Middle/Upper Eocene boundary in Egypt. Middle East Research Center, Ain Shams University, Earth Science Series. 1994;8:210-233.

8. Anan HS. Accelerated evolution in representatives of the genera Orthokarstenia and Discorbis (Benthic foraminifera) in the Maastrichtian and Paleocene of Egypt (Misr). Neues Jahrbuch für Geologie und Paläontologie Monatshefte; 1998. 365-375 p.

9. Anan HS. Paleocene Vaginulininae (benthic foraminifera) of Duwi section, Red Sea coast, Egypt. Egyptian Journal of Paleontology. 2001;1:135-139.

10. Anan HS. Stratigraphy and paleobiogeography of some Frondiculariinae and Palmulinae benthic foraminiferal general in the Paleocene of Egypt (Misr). Neues Jahrbuch für Geologie und Paläontologie Monatshefte; 2002a. 629-640 p.

11. Anan HS. Two new benthic foraminiferal species from the Maastrichtian and Paleocene rocks of northern Egypt. Middle East Research Center, Ain Shams University, Earth Science Series. 2002b;16:141-144.

12. Anan HS. Paleontological and biostratigraphical remarks on some diagnostic Tethyan benthic foraminifera. 2nd International Conference on the Geology of the Tethys. Giza: Cairo University Press; 2007a. 303-308 p.

13. Anan HS. Maastrichtian-Paleogene LeRoy's benthic foraminiferal species from Egypt and Tethyan-Atlantic regions. Revue de Paléobiologie. 2008;27(2):357-376.

14. Anan HS. Paleontology, paleogeography, paleoenvironment and stratigraphical implications of the Nakkady's benthic foraminiferal fauna in Egypt and Tethys. Egyptian Journal of Paleontology. 2009b;9:31-52.

15. Anan HS. Early Eocene Ellipsoglandulina arafati n. sp. (benthic foraminifera) from Abu Zenima section, west central Sinai, Egypt. Egyptian Journal of Paleontology. 2009c;9:111-117.

16. Anan HS. Lenticulina ennakhali n. sp. (benthic foraminifera) from the Paleocene-Early Eocene succession of Abu Zenima section, west central Sinai, Egypt (Misr). Al-Azhar University Journal, Natural Sciences Series. 2010c;12:19-22.

17. Anan HS. Paleontology, paleoenvironments, palaeogeography and stratigraphic value of the Maastrichtian-Paleogene and Recent foraminiferal species of Anan in the Middle East. Egyptian Journal of Paleontology. 2011;11:49-78.

18. Anan HS. Paleontology, paleoenvironment and stratigraphic value of the Maastrichtian-Paleogene benthic foraminifera of Said \& Kenawy (1956) from Egypt and Tethys. Egyptian Journal of Paleontology. $2012 \mathrm{a} ; 12: 17-30$ 
19. Anan HS. A lineage phylogeny from some Cretaceous-Tertiary agglutinated benthic foraminiferal species in Egypt and Tethys. Egyptian Journal of Paleontology. 2012b;12:59-72.

20. Anan HS. Foraminiferal taxa dedicated by the Egyptian paleontologist Saad Ansary. Egyptian Journal of Paleontology. 2013a;13:49-59.

21. Anan HS. Significant episodes of tectonic activities in the Paleogene of Al Ain area, United Arab Emirates. International Journal of Chemical and Natural Science. 2014a;2(6):180-188.

22. Anan HS. Review on spatio-temporal distribution of three benthic foraminiferal species from Egypt and Tethys. Egyptian Journal of Paleontology. 2014b;14:65-72.

23. Anan HS. Paleogene Lagenid Percultazonarias (Foraminifera) in Egypt: paleontology, stratigraphy, paleogeography and some taxonomical considerations. Egyptian Journal of Paleontology. 2015a;15:13-30.

24. Anan HS. Evolutionary lineage of the Maastrichtian Bolivinoides draco group (benthic foraminifera) in Abu Zenima section, west central Sinai, Egypt. Arabian Journal of Geosciences. 2017a;10:431.

25. Anan HS. Paleontology and paleogeography of the Paleogene benthic foraminiferal species of Plummer in Egypt and other Atlantic-Tethyan regions. Journal of Tethys. 2017b;5(3):272-296.

26. Anan HS. Contribution to the paleontology, stratigraphy and paleobiogeography of some diagnostic Pakistanian Paleogene foraminifer in the Middle East. Earth Sciences Pakistan. 2019a;3(1):29-34.

27. Anan HS. On the variability of benthic foraminiferal species of the genus Pleurostomella in the Tethys. . 2019b;7(3):173-181.

28. Aly HA, Abd El-Aziz SM, Abd El-Gaied IM. Middle and Upper Eocene benthic foraminifera from Wadi Bayad El Arab-Gebel Homret Shaibon area, Northeastern Beni Suef, Nile Valley, Egypt. Egyptian Journal of Paleontology. 2011;11:79-131.

29. Futyan AI. Late Mesozoic and Early Cainozoic benthonic foraminifera from Jordan. Palaeontology. 1976;19(3):53-66.

30. Hasson PH. New observations on the biostratigraphy of the Saud Arabian Umm er Radhuma Formation (Paleogene) and its correlation with neighboring regions. Micropaleontology. 1985;31(4):335-364.

31. Hewaidy AA, Al-Hitmi H. Cretaceous-Early Eocene biostratigraphy of the Dukhan oil field, west Qatar, Arabian Gulf. Middle East Research Center, Ain Shams University, Earth Science Series. 1994;8:1-24.

32. Anan HS. Three new species of benthic foraminifera from the Middle Upper Eocene of Jabal Hafit, Al Ain area, United Arab Emirates. Neues Jahrbuch für Geologie und Paläontologie Monatshefte; 2003. 529-536 p.

33. Anan HS. Taxonomy, paleogeography, paleoecology and paleoclimatology of the Late Eocene benthic foraminifera of Jabal Malaqet, United Arab Emirates, northern Oman Mountains. Egyptian Journal of Paleontology. 2007b;7:67-85.

34. Anan HS. Paleontology and stratigraphical distribution of suborder Lagenina (benthic foraminifera) from the Middle-Late Eocene Mazyad Member of the Dammam Formation in Jabal Hafit, Al Ain area, United Arab Emirates, Northern Oman Mountains. Revue de Paléobiologie. 2009a;28(1):1-18

35. Anan HS. Paleontology and stratigraphical distribution of suborder Rotaliina (benthic foraminifera) from the Middle-Late Eocene Mazyad Member of the Dammam Formation in Jabal Hafit (Al Ain area), United Arab Emirates, Northern Oman Mountains. Revue de Paléobiologie. 2010a;29(1):157-184

36. Anan HS. Lenticulina ennakhali $n$. $s p$. (benthic foraminifera) from the Paleocene-Early Eocene succession of Abu Zenima section, west central
Sinai, Egypt (Misr). 1st International Conf. for Basic and Applied Sciences (ICBAS): Gaza, Palestine; 2010. 19-22 p.

37. Anan HS. Paleocene Lagenid benthic foraminifera of Jabal Mundassa, Al Ain Area, United Arab Emirates. Egyptian Journal of Paleontology. 2015b;15:61-83.

38. Haque AFMM. The foraminifera of the Ranikot and the Laki of the Nammal Gorge, Salt Range, Pakistan. Pakistan Geological Survey Memoir, Palaeontologica Pakistanica; 1956. 229 p.

39. Haque AFMM. Some middle to late Eocene smaller foraminifera from the Sor Rang, Quetta District, West Pakistan. Pakistan Geological Survey Memoir, Palaeontologica Pakistanica. 1960;2(2):9-57.

40. Haque AFMM. The smaller foraminifera of the Meting Limestone (Lower Eocene) Meting, Hyderabad Division, West Pakistan. Karachi: Govt. of Pakistan Press; 1959. 43 p.

41. Nomura R, Brohi IA. Benthic foraminiferal fauna during the time of the Indian-Asian contact, in southern Balochistan, Pakistan. Marine Micropaleontology. 1995;24:215-238.

42. Loeblich A, Tappan H. Foraminiferal genera and their classification. Van Nostrand Reinhold (VNR);1988.

43. Ehrenberg CG. Invisible calcareous piebalds and pebbles as the main constituents of the chalk glacier. Report on the Appropriate Negotiations of the Royal Prussian Academy of Sciences in Berlin; 1838. 192-200 p.

44. Risso A. Natural History of the Principal Productions of Southern Europe and particularly those of the Surroundings of Nice and the Maritime Alps. Paris: F.G. Levrult; 1826.

45. Orbigny A. Physical, political and natural history of the island of Cuba. Paris: Arthus Bertrand; 1839. 224 p.

46. Ali MY. Micropaleontological and stratigraphical analyses of the Late Cretaceous/Early Tertiary succession of the Southern Nile Valley (Egypt). Ruhr University Bochum; 2003. 1-197p.

47. Loeblich AR, Tappan H. Some new and revised genera and families of hyaline calcareous Foraminiferida (Protozoa). Transactions of the American Microscopical Society. 1986;105:239-265.

48. Loeblich AR \& Tappan H. A revision of some Glanduline Nodosariidae (Foraminifera). Smithsonian Miscellaneous Collections; 1955. 9 p.

49. Boomgaart L. Smaller Foraminifera from Badjonegoro (Java). Sappemeer: Smit \& Dontje; 1949.

50. Reuss AE. New foraminifera from the strata of the Austrian Terriär basin Memorandum of the Imperial Academy of Sciences, Mathematical and Natural Sciences class; 1850. 465-390 p.

51. Ansary SE. A new foraminiferal fauna from the Upper Egypt. Bulletin of Faculty of Engineering, Cairo Univ Press; 1954. 305-334 p.

52. Fornasini C. Quinto contributo alla conoscenza della microfauna Terziaria Italiana. Memorie della R. Accademie della Scienze dell'Istituto di Bologna, Scienze Naturali, ser. 1894;5(4):201-230.

53. Hewaidy AA, Strougo A. Maastrichtian-lower Eocene benthic foraminiferal distribution and paleoecology of three outcrop sections in Farafra. Egyptian Journal of Paleontology. 2001;1:1-22.

54. Reuss AE. The foraminifera of the Westphalian Chalk Formation. Session Reports of the Imperial Academy of Sciences in Vienna, Mathematical and Natural Sciences class. 1860;40:147-238.

55. Defrance JLM. Natural Science Dictionary. Vol 35. Paris: FG Levrault; 1826.

56. Defrance JLM. Natural Science Dictionary. Vol 32. Strasburg: EG Levrault; 1824. 
57. Ismail AA. Late Cretaceous-Early Eocene benthic foraminifera from Esh El Mallaha area, Egypt. Revue de Paléobiologie. 2012;31(1):15-50.

58. Luger P. Stratigraphy of the marine Cretaceous and Late Tertiary in the southwestern Obernil Basin (SW-Egypt) under special consideration of micropaleontology, palaeology and paleogeography. Berlin geoscientific essays (A). 1985;63:1-151.

59. Youssef M, Taha S. Biostratigraphy and Paleoecology of Paleocene/ Eocene $(\mathrm{P} / \mathrm{E})$ interval of some geological sections in Central Egypt. Arabian Journal of Geosciences. 2012;6(11):4279-4298.

60. Mac Fadyen WA. Foraminifera from the Green Ammonite Bds, lower Lias, of Dorset. Philosophical Transaction of the Royal Society B. 1941;231 (576):1-73.

61. Berthelin G. Foraminifera of the middle Lias of the Vendée. Magazine and Zoology Shop Pure and Applique, Paris. 1879;3(7):24-41.

62. Cushman JA. An outline of the re-classification of the Foraminifera Contributions from the Cushman Laboratory for foraminiferal Research. 1927;3:1-105.

63. Neugeboren JL. Foraminifera of Felsö-Lapugy, second article Negotiations and communications of the Transylvanian Association for Natural Sciences to Sibiu. 1850;1:118-127.

64. Reuss AE. The foraminifera of the Westphalian Chalk Formation. K Akad Vienna math Naturw CI Sitzungsber: Vienna.1860; 40:147-238.

65. Chapman F, Barr WJ, Collins AC. Tertiary foraminifera of Victoria, Australia-The Balcombian deposits of Port Phillip, part 3. Journal of the Linnaean Society of London, Zoology. 1934;38:553-577.

66. Lamarck JB. Continued memories of fossils around Paris. Annals Museum Natural History Nation. 1804;5:179-188.

67. Hussein AI. Bulimina jacksonensis Cushman from the Mokattamian Stage (Middle and Upper Eocene) of Egypt and its biostratigraphic significance. Middle East Research Center, Ain Shams Univ, Earth Science Series. 1992;6:107-113.

68. Abul-Nasr RA. Middle-Upper Eocene benthic foraminifera of Wad Tayiba and Wadi Bagha (western Sinai): A comparative study. Middle East Research Center, Ain Shams University, Earth Science Series, Cairo. 2000;14:49-76.

69. Silvestri A. Structural research on some forms of Trubi di Bonfornella (Palermo). Memories of the Pontifical Roman Academy of the New Lincei. 1904;22:235-276.

70. Goës A. A synopsis of the Arctic and Scandinavian Resent marine Foraminifera hitherto discovered. Kongl Swedish Academy of Sciences Actions. 1894;25(9):1-27.

71. Cushman JA. The foraminifera of the Atlantic Ocean, Part 4, Lagenidae Bulletin United States National Museum. 1923;104(4):1-228.

72. Hussein AI. Middle Eocene palmate benthonic foraminifera from the Nile Valley, and their paleoecological significance. Middle East Research Center, Ain Shams University, Earth Science Series. 1998;12:214-218.

73. Brotzen F. The Swedish Paleocene and its foraminiferal fauna. Sweden Geological Survey of Sweden; 1948. 493:1-140.

74. Aref M, Youssef M. Benthic foraminiferal biostratigraphy of the Late Cretaceous/Early Tertiary succession along the Red Sea coastal area, Egypt. 3rd International Conference of the Geology of Arab World, Cairo University; 1996. 539-573 p.

75. Plummer HJ. Foraminifera of the Midway Formation in Texas. Bulletin University of Texas; 1927. 3-206 p.

76. Saidova KhM. O sovremennom sosttoyanii sidtemy nadvidovykh taksonov Kaynozoyskikh bentosnykh foraminifer. Institut Okeanologii P. P. Shirshova. Akademiya Nauk SSSR. 1981;73 p.
77. Lea I. Contributions to Geology. Philadelphia: Carey, Lea \& Blanchard; 1833.

78. Helal SA. Contribution to the Eocene benthic foraminifera and ostracoda of the Fayoum Depression, Egypt. Egyptian Journal of Paleontology. 2002;2:105-155.

79. Anan HS, Hewaidy A. Biostratigraphy and distribution of the Paleocene benthonic foraminifera in the Nile Valley Facies in Egypt. Middle East Research Center, Ain Shams University, Earth Science Series. 1986;6:1-32.

80. Aubert J, Berggren WA. Paleocene benthonic foraminiferal biostratigraphy and paleoecology of Tunisia. Bulletin Centre de Recherches Pau-SNPA. 1976;10(2):379-469.

81. Anan HS, Sharabi SA. Benthonic foraminifera from Upper CretaceousLower Tertiary rocks of northwest Kharga Oasis, Egypt. Middle East Research Center, Ain Shams University, Earth Science Series. 1988;2:191-218.

82. Wedekind PR. Introduction to the foundations of historical geology. Volume II Microbiostratigraphy the coral and foraminifera period. Stuttgart: Ferdinand Enke; 1937.

83. Denys de Montfort P. Systematic Conchyliology and Methodical Classification of Shells. Paris: Schoell; 1808.

84. Anan HS. Maastrichtian-Paleocene micropaleontology and biostratigraphy of Qarn El Barr section, Al Dhayd area, United Arab Emirates. Al-Azhar Bulletin of Science, Al-Azhar University. 1993b;4(2):639-670.

85. Stache G. The foraminifera of the tertiary marl of Whaingaroa Port (Prov. Auckland), Novara Expedition, 1857-1859. Geologische Theil. 1864;1(2):159-304.

86. Hewaidy AA, Farouk S, EL-Balkiemy AF. Foraminiferal Biostratigraphy, Stages Boundaries and Paleoecology of the Uppermost MaastrichtianLower Eocene Succession at Esh El-Mellaha Area, North Eastern Desert, Egypt. Journal of American Science. 2017;13(5):74-113.

87. Silvestri A. Structural research on some forms of Trubi di Bonfornella (Palermo). Memories of the Pontifical Roman Academy of the New Lincei. 1904;22:235-276.

88. Silvestri A. Pliocene Foraminifers of the Province of Siena, Part 2. Memories of the Pontifical Academy of the New Lincei, Rome. 1898; $15: 155-381$.

89. Brady HB. Report on the foraminifera dredged by HMS Challenger, during the years 1873-1876 (in Report on the Scientific Results of the Voyage of the HMS Challenger Zoology Vol 9). Unknown Binding; 1884.

90. Jones TR. A guide to the examination and investigation of the structure and nature of microscopic objects (in Griffith JW, Henfrey A. Eds.). The Micrographic Dictionary: Van Voorst, London; 1875. 316-320 p.

91. Loeblich AR, Tappan H. Supergeneric calassification of the Foraminiferida (Protozoa). Micropaleontology. 1984;30:1-70.

92. Orbigny AD d' Methodical table of the cephalopod class. Annals of Natural Sciences, Paris. 1826;7:245-314.

93. Glaessner MF. Studien über Foraminiferen aus der Kreide und dem Tertiär des Kaukasus. I. Die Foraminifern der Ältesten Tertiärschichten des Nordwest-Kaukasus. Problemy Paleontologii, Paleontologicheskava Laboratotiya Moskovskogo Gosudarstvennogo Universiteta. 1937;23:349-410.

94. Klasz I de, Rérat D. Quelques nouveaux foraminiféres du Crétacé et du Tertiaire du Gabon (Afrique Équatoriale). Revue de Micropaléontologie. 1962;4:175-189.

95. Shahin A. Biostratigraphic significance, paleobiogeography and paleobathymetry of Tertiary Buliminacea and Bolivinacea in the 
Western Sinai, Egypt. Neues Jahrbuch für Geologie und Paläontologie, Abh. 2000;216(2):195-231.

96. Marsson Th. The foraminifera of the white chalk of the islands Rügen Mitteilugen of the scientific association for new-Vorpommern and Rugen in Greifswald. 1878;10:115-196.

97. Loeblich AR, Tappan H. Six new generic names in the Mycetozoida (Trichiidae) and Foraminiferida (Fischerinidae, Buliminidae, Caucasinidae, and Pleurostomellidae) and a redescription of Loxostomum (Loxostomidae, new family). Proceedings of the Biological Society of Washengton. 1962;75:107-113.

98. Finlay HJ. New Zealand foraminifera: Key species in stratigraphy, 3 Transactions of the Royal Society of New Zealand. 1939;69:309-329.

99. Tjalsma RC, Lohmann GP. Paleocene-Eocene bathyal and abyssa benthic foraminifera from the Atlantic Ocean. Micropaleontology, Special Publication. 1983;4:1-90.

100. Cushman JA. Eouvigerina a new genus from the Cretaceous. Contributions from the Cushman Laboratory for foraminiferal Research 1926;2:3-6.

101. Keller G. Biotic turnover in benthic foraminifera across the Cretaceous/Tertiary boundary at El Kef, Tunisia. Palaeogeography, Palaeoclimatology, Palaeoecology. 1988;66(3-4):153-171.

102. Culver SJ. Benthic foraminifera across the Cretaceous-Tertiary (K-T) boundary: a review. Marine Micropaleontology. 2003;47(3-4):177-226.

103. Cushman JA, Wickenden TD. Anew foraminiferal genus from the Upper Cretaceous. Contributions from the Cushman Laboratory for Foraminiferal Research. 1928;4(1):12-13

104. Speijer RP, Van Der Zwaan GJ, Schmitz B. The impact of Paleocene/Eocene boundary events on muddle neritic benthic foraminiferal assemblages from Egypt. Marine Micropaleontology. 1996;28(2):99-132.

105. Alegret L, Ortiz S, Arenillas I, et al. Paleoenvironmental turnover across the Paleocene/Eocene boundary at the Stratotype section in Dababiya (Egypt) based on benthic foraminifera. Terra Nova. 2005;17(6):526-536

106. Andreae A. Contribution to the knowledge of the Alsatian Tertiary, part 2-The Oligocene strata. Treatises on the Special Geological Map of AlsaceLothringen, Strasbourg. 1884;2(3):1-239.

107. Reiss Z. Reclassification of perforate foraminifera. Bulletin of the Geological Survey of Israel. 1963;35:1-111.

108. Howe HV, Wallace WE. Foraminifera of the Jackson Eocene at Danville Landing on the Ouachita, Catahoula Parish, Louisiana. Bulletin of the Geological Survey of Louisiana.1932;2:1-118

109. Saidova KhM. On the current state of the Sydteme of supraspecific taxa of the Cenozoic benthic foraminifera. Institute of Oceanology. Academy of Sciences of the USSR: PP Shirshova; 1981.73 p.

110. Dietrich WO. On the stratigraphy of the Colombian Eastern Cordillera. Central Journal of Mineralogy, Geology and Paleontology; 1935. 74-82 p.

111. Karsten H. About the geognostic conditions of western Colombia. The republics of New-Granada and Equador. Official Report 32 Meeting of German Naturalists and Doctors on Vienna; 1858. 80-117 p.

112. Anan HS. Paleocene benthonic foraminifera of Jabal Malaqet, Al Ain region, United Arab Emirates. Al-Azhar Bulletin Science, Al Azhar University: Cairo. 1993a;4(1):293-320.

113. Sztrákos K. Eocene foraminifers in the Adour Basin (Aquitaine, France): biostratigraphy and taxonomy. Revue de Micropaléontologia. 2000;43(1):71-172
114. Ortiz S, Thomas E. Lower-middle Eocene benthic foraminifera from the Fortuna Section (Betic Cordillera, southeastern Spain). Micropaleontology. 2006;52(2):97-150.

115. Alegret L, Ortiz S. Global extinction event in benthic foraminifera across the Paleocene/Eocene boundary at the Dababiya Stratotype section. Micropaleontology. 2007;52(5):433-447.

116. Boltovskoy E, Vera Ocampo J. Benthic foraminifers from DSDP site 219 (Eocene-Pleistocene, Arabian Sea). Revista Espaňola de Micropaleontologia. 1993;25(1):127-156.

117. Speijer RP. Extinction and recovery patterns in benthic foraminifera paleocommunities across the Cretaceous/Paleogene and Paleocene/ Eocene boundaries. Geologica Ultraiectina. 1994;124:1-191.

118. Cherif OH, Al-Rifaiy IA, El Deeb WZ. "Post-Nappes" early Tertiary foraminiferal paleoecology of the northern Hafit area, south of Al Ain City (United Arab Emirates). Micropaleontology. 1992;38(1):37-56.

119. Haeckel E. Systematic phylogeny: Draft of a Natural System of Organisms on the Basis of Their History. Part I, Systematic Phylogeny of Protists and Plants. Berlin: Georg Reimer; 1894

120. Galloway JJ. A manual of foraminifera. Bloomington: Principia Press; 1933.

121. Cushman JA. The foraminifera of the Atlantic Ocean, Part 4, Lagenidae Bulletin United States National Museum. 1923;104(4):1-228.

122. Berggren WA. Late Paleocene-Early Eocene benthonic foraminifera bio- stratigraphy and Paleoecology of Rockall Bank. Micropaleontology. 1974a;20(4):426-448.

123. Loeblich AR, Tappan $H$. The status of the foraminifera genera Ammodiscus Reuss and Involutina Terquem. Micropaleontology. 1961;7(2):189-192.

124. Reuss AE. The foraminifera of the Westphalian Chalk Formation. Session Reports of the Imperial Academy of Sciences in Vienna, Mathematical and Natural Sciences class. 1860;40:147-238.

125. Silvestri A. Sur genus Ellipsoglandulina, Acts and Rendiconti $R$. Academy of Sciences, Letters and Art of the Zealous; 1901.

126. Rzehak A. About some strange foraminifera from the Austrian Tertiary. Annals of Natural History Museum, Vienna. 1895;10(2):213-230.

127. Gümbel CW. Contributions to the foraminifera fauna of the Northern Alpine Euclidae formations. Treatises of the Royal Bayayer Academy of Sciences, class. Munich. 1870;10(2):581-730.

128. Ismail AA. Some Upper Cretaceous benthonic foraminifera from Sufr E Dara, Eastern Desert, Egypt. Egyptian Journal of Geology. 1992;35(12):221-243

129. Reuss AE. Above the fossil foraminiferous and entomostraceen of the Septarienthone of the neighborhood of Berlin. Journal of the German Geological Society, Berlin. 1851;3:49-92.

130. Al-Hitmi H. Foraminiferal biostratigraphy of the Upper Cretaceous succession in southwest Qatar, Arabian Gulf. Egyptian Journal of Paleontology. 2002;2:41-54.

131. Hofker J. Recent Peneroplidae. Pt.1 (continued). Journal of the Royal Microscopical Society. 1951;71:223-239.

132. Montfort P Denys. Systematic Conchyliology and Methodical Classification of Shells. Paris: Schoell; 1808.

133. Fichtel L von, von Moll JPC. A microscope, shells, and other small, and the Nautilus of genets Aragonauta, to the nature of the painted and described. Vienna: Degen; 1798

134. Orbigny AD. Foraminifères fossils du Bassin Tertiary de Vienne (Autriche). Paris: Gide et Comp;1846. 
135. Speijer RP. Systematics and paleoecology of the foraminifer Neoeponides duwi (Nakkady) from the Paleocene of Egypt. Micropaleontology. 2003;49(2):146-150.

136. Sigal J. Stratigraphic Overview of Cretaceous Micropaleontology. In 19th International Geological Congresses, Regional Monorraphies, Series I: Algeria. 1952;26:1-47.

137. Thalmann HE. Bibliography and index to new genera, species and varieties of foraminifera for the year 1936. Journal of Paleontology. 1939;13(4):425-365.

138. Brady HB, Parker WK, Jones TR. On some foraminifera from the Abrohlos bank. Transactions of the Zoological Society of London. 1890;12(7):211-239.

139. Saperson E, Janal, M. Biostratigraphy of the Anomalinidae and Cibicididae in Soviet Tethyan Paleogene. Micropaleontology. 1980;26(4):392-413.

140. Sztrákos K. Paleocene and lowest Eocene foraminifera from the north Pyrenean trouph (Aquitaine, France). Revue de Micropaléontologia. 2005;48(3):175-236.

141. Galeotti S, Coccioni R. Changes in coiling direction of Cibicidoides pseudoacutus (Nakkady) across the Cretaceous-Tertiary boundary of Tunisia: palaeoecological and biostratigraphic implications. Palaeogeography, Palaeoclimatology, Palaeoecology. 2002;178(34):197-210

142. Guasti E, Kouwenhoven TJ, Brinkhuis H, et al. Paleocene sea-level and productivity changes at the southern Tethyan margin (El Kef, Tunisia). Marine Micropaleontology. 2005;55(1-2):1-17.

143. Ernst SR, Guasti E, Dupuis C, et al. Environmental perturbation in the southern Tethys across the Paleocene/Eocene boundary (Dababiya, Egypt): Foraminiferal and clay mineral records. Marine Micropaleontology. 2006;60(1):89-111.

144. Schwager C. Framework of the proposed classification system for shelled foraminifera. Bolletina R, Geological Committee of Italy. 1877;8:18-27.

145. Bermúdez PJ. Systematic study of rotaliform foraminifers. Bolivian Geology Venezuela. 1952;2(4):1-230.

146. Hofker J. Über die Familie Epistomariidae (Foram). Palaeontographica. 1954;15A:166-206.

147. Galloway JJ. A manual of foraminifera. Bloomington: Principia Press; 1933.

148. Parker WK, Jones TR. On some foraminifera from the North Atlantic and Arctic Oceans, including Davis Straits and Baffin's Bay. Philosophical Transactions of the Royal Society. 1865;155:325-441.

149. Hasson PH. New observations on the biostratigraphy of the Saudi Arabian Umm er Radhuma Formation (Paleogene) and its correlation with neighboring regions. Micropaleontology. 1985;31(4):335-364.

150. Singh SN, Kalia P. A new asterigerinid genus from the Kirthars of Rajasthan, India. Geophylogeny. 1972;1(2):156-160.

151. Uchio T. Foraminiferal assemblage from Hachijo Island, Tokyo Prefecture, with descriptions of some new genera and species. Japanese Journal of Geology and Geography. 1952;22:145-159.

152. Schultze MS. Über den Organismus der Polythalamien (Foraminiferen), nebst Bermerkungen über die Rhizopoden im Allegemeinen. Leipzig: Wilhelm Engelmann; 1854.

153. Farouk S. On the occurrence of a Late Eocene 'Turborotalia cerroazulensis cocoaensis Zone' around Gabal Libni, Northern Sinai, Egypt. Journal of Paleontology. 2007;7:59-66.

154. Brady HB. Notes on some of the reticularian Rhizopoda of the 'Challenger' Expedition, part 3. 1. Classification. 2. Further notes on new species. 3. Note on Biloculina mud. Quaternary Journal of Microscopical Science, new series. 1881;21:31-71.

155. Toulmin LD. Eocene smaller foraminifera from the Salt Mountain Limestone of Alabama. Journal of Paleontology. 1941;15:567-611.

156. Loeblich AR, Tappan H. Sarcodina chiefly "Thecamoebians" and Foraminiferida. In: Moore RC. (Ed). Treatise on Invertebrate Paleontology. America and University of Kansas Press; 1964.

157. Brotzen F. Flintrännaus and Trindelrännans geology (Öresund). Sweden's Geological Survey: Stockholm. 1940;34:3-33.

158. Gonzáles-Donoso JM. New data on texture and test structure of some foraminifers in the Granada Basin (Spain). Micropaleontology Review. 1969;12:3-8.

159. Brotzen, F. The Foraminiferengattung Gavelinella nov Gene and the Rotaliform System. Yearbook Sverringe's Geological Survey. 1942;36(8):1-60.

160. Hofker J. Tertiary foraminifera of coastal Ecuador: Part II, Additional notes on the Eocene species. Journal of Paleontology. 1956;30:891-958.

161. Brotzen F. The Foraminiferengattung Gavelinella nov Gene and the Rotaliform System. Yearbook Sverringe's Geological Survey. 1942;36(8):1-60.

162. Anan HS. A lineage phylogeny for some Maastrichtian to Ypresian benthic foraminifera in Egypt. Egyptian Journal of Paleontology. 2004:4:39-57.

163. Hofker J. Foraminifera of the Upper Cretaceous of Northwest Germany and Holland. Supplements to the Geological Yearbook. 1957;27:1-464.

164. Ehrenberg CG. About the formation of chalk cliffs and the chalk gel by invisible organisms. Physical Treatises of the Royal Academy of Sciences in Berlin; 1838. 59-147 p.

165. Anan HS. Early Eocene foraminifera of Jabal Hafit, United Arab Emirates. Middle East Research Center, Ain Shams University, Earth Science Series. 1996;10:147-162.

166. Anan HS. Foraminiferal taxa dedicated by the Palestenian paleontologist Hamed El-Nakhal. Egyptian Journal of Paleontology. 2013b;13:109-120.

167. Hewaidy AA. Biostratigraphy of the Umm Er Radhuma Formation in South-East Qatar, Arabian Gulf. New Yearbook of Geology and Paleontology.1994;193(2):145-164.

168. Haynes J, Nwabufo-Ene K. Foraminifera from the Paleocene phosphate beds, Sokoto, Nigeria. Revista Española de Micropaleontología. 1998;30(2):51-76

169. Reiss Z. Reclassification of perforate foraminifera. Bulletin of the Geological Survey of Israel. 1963;35:1-111.

170. Le Calvez Y. Revision of the Lutheran Foraminifers of the Paris Basin 2: Otaiidae and Affine Families. Memoirs of the Detailed Geological Map Service of France; 1949. 1-54 p.

171. Terquem O. The foraminifers of the Eocene of the envorons of Paris. Memoirs of the Geological Society of France. 1882;3(2):1-193.

172. Cushman JA. New genera and species of the families Verneuilinidae and Vulvulinidae and of the subfamily Virgulininae. Cushman Foundation for Foraminiferal Research, Special Publication. 1936;6:1-71.

173. Parker WK, Jones TR, Brady HB. Contributions to the knowledge of the foraminifera-On the Rhizopodal fauna of the Shetlands. Transactions of the Linnean Society of London. 1864;24:463-476.

174. Berggren WA. Paleocene benthonic foraminiferal biostratigraphy, biogeography and paleoecology of Libya and Mali. Micropaleontology. 1974b;20(4):449-465. 
175. Berggren WA. Micropaleontology and Cenozoic paleoclimatology, part II: 277-299 (in Berggren and Phillips: Influence of the Continental drift on the distribution of the Tertiary benthic foraminifera in the Caribbean and Mediterranean regions), In: Gray C. (Ed.): Symposium on the geology of Libya. Faculty of Science, University of Libya: Tripoli; 1971. 263-299 p

176. Mintz LW. Historical Geology, the Science of a Dynamic Earth. In: The fountainhead of Stratigraphy: The Mesozoic Erathem. 3rd Ed. Merrill Publication Company: USA; 1981. 434-502.

177. Rosenbaum G, Lister GS, Duboz C. Relative motions of Africa, Iberia and Europe during Alpine orogeny. Tectonophysics. 2002;359(12):117-129.

178. Haq BU, Aubry MP. Early Cenozoic calcareous nannoplankton biostratigraphy and palaeobiogeography of North Africa and the Middle East and Trans-Tethyan correlations. 2nd Symposium of Geology of Libya; 1978. 271-304 p.

179. Anan HS. Late Eocene biostratigraphy of Jabals Malaqet and Mundassa of Al Ain region, United Arab Emirates. Revue de Micropaléontologie. 1995;38(1):3-14.

180. Rögl F. Mediterranean and Paratethys. Facts and hypotheses of an Oligocene to Miocene paleogeography (short overview). Geologica Carpathica. 1999;50(4):339-349.
181. Meulenkamp JE, Sissingh W. Tertiary paleogeography and tectonostratigraphic evolution of the Northern and Southern PeriTethys platforms and the intermediate domains of the African-Eurasian convergent plate boundary zone. Palaeogeography, Palaeoclimatology, Palaeoecology. 2003;196(1-2):209-228.

182. Berggren WA, Perason PN. A revised tropical to subtropical Paleogene planktonic foraminiferal zonation. Journal of Foraminiferal Research. 2005;35(4):279-298.

183. Anan HS. Early Eocene foraminifera of Jabal Hafit, United Arab Emirates. Middle East Research Center, Ain Shams University, Earth Science Series: Cairo. 1996;10:147-162.

184. Berggren WA, Aubert J. Paleocene benthonic foraminiferal biostratigraphy, paleobiogeography and paleoecology of AtlanticTethyan regions: Midway-type fauna. Palaeogeography, Palaeoclimatology, Palaeoecology. 1975;18(2):73-192.

185. Cherif OH, El Deeb WZ. The Middle Eocene-Oligocene of the Northern Hafit Area, south of Al Ain City (U.A.E.). Geologie Méditerranéenne. 1984;11(2):207-217.

186. Anan HS, Hamdan AR. Bolivinoides curtus Reiss from the Paleocene of Jabal Malaqet, East of Al Ain, west of the Northern Oman Mountains, United Arab Emirates. Journal of Faculty of Science, United Arab Emirates University. 1992;4(1):200-211. 